\title{
Some Combinatorial and Geometric Constructions of Spherical Buildings
}

\author{
Hendrik Van Maldeghem and Magali Victoor
}

\begin{abstract}
We survey some known and new combinatorial and geometric constructions of the Lie incidence geometries related to some spherical buildings, in particular of some exceptional types.
\end{abstract}

\section{Contents}

1 Introduction 2

2 Definitions-Spherical Buildings and Lie Incidence Geometries 2

2.1 Coxeter systems and Coxeter complexes . . . . . . . . . . . . 2

2.2 Buildings and spherical buildings . . . . . . . . . . . . . 4

2.3 Graphs, embeddings and more conventions and notation . . . . . . . 7

2.3.1 Graphs . . . . . . . . . . . . . . . . . 7

2.3.2 Embeddings ................... 8

3 Intersections of Quadrics $\quad 8$

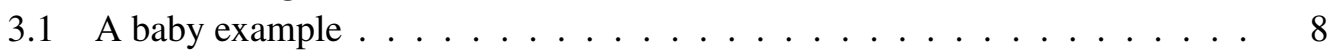

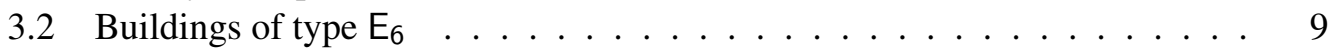

3.3 Buildings of type $E_{7} \ldots \ldots \ldots \ldots \ldots$

4 Equator and Trace Geometries 13

4.1 The general principle and some easy examples . . . . . . . . . . . . . . . . 13

4.2 Two examples related to $\mathrm{F}_{4} \ldots \ldots \ldots \ldots \ldots$

4.3 Examples related to $\mathrm{E}_{6} \ldots \ldots \ldots \ldots \ldots \ldots$

4.4 Examples related to $E_{7} \ldots \ldots \ldots \ldots \ldots$

4.5 Examples related to $E_{8} \ldots \ldots \ldots \ldots$

5 Projective Constructions 16

5.1 Hyperbolic polar spaces and half spin geometries . . . . . . . . . . . . . 17

5.2 Two constructions of the Segre variety $\mathscr{S}_{2,2}(k) \ldots \ldots \ldots$

5.3 A line Grassmannian . . . . . . . . . . . . . . . . . . . . . . . . 19

5.4 The Cartan variety . . . . . . . . . . . . . . . . 20

6 Combinatorial Constructions $\quad 20$

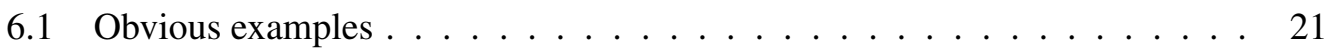

6.2 The line Grassmannian $A_{5,2}(k)$ again . . . . . . . . . . . . . . . . . 21

6.3 Metasymplectic spaces and $\mathrm{E}_{6,1}(k) \ldots \ldots \ldots \ldots 22$ 
7 Coxeter Complexes and associated Graphs 2

7.1 Apartments of type $\mathrm{E}_{6} \ldots \ldots \ldots \ldots \ldots \ldots \ldots$

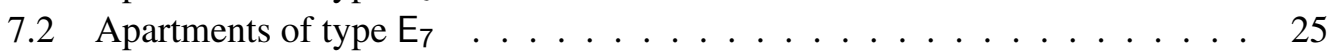

7.3 Apartments of type $\mathrm{E}_{8} \ldots \ldots \ldots \ldots \ldots \ldots$

7.4 Apartments of type $\mathrm{F}_{4} \ldots \ldots \ldots \ldots \ldots \ldots$

8 Conclusion and open problems $\quad 30$

\section{Introduction}

The current paper grew out of a geometric approach to the Freudenthal-Tits Magic Square. This Square is most popular in the area of Lie algebras and algebraic geometry. However, Tits [6] introduced an incidence geometric version of the square in his habilitation thesis, ten years before he gave a general formula for the Lie algebras appearing in the Square. These incidence geometries are now generally known as instances of Lie incidence geometries.

Our geometric approach focuses on characterizations of the point-line geometries of the Square and their embeddings into projective space. During this study a lot of natural geometric and combinatorial connections between the cells of the Square became apparent. This paper reports on these connections as far as constructions are concerned. However, also other types of Lie incidence geometries are involved, and certain generalizations lead to constructions of classes of Lie incidence geometries that have no direct connection anymore with the Square.

We present four types of constructions, many of them disposing links between Lie incidence geometries. Each time we mention which cells of the Square are involved, but we also give many examples outside the Square. A systematic treatment from this viewpoint of the Square is beyond the scope of this paper, and would lead us too far. Instead, we will present our constructions as examples of geometries related to the square.

In the final section we present some constructions of Coxeter complexes related to the exceptional types. This implies alternative constructions of some well-known graphs like some Johnson graphs and the Gosset graph. These constructions are mainly an application of the section about equator and trace geometries.

\section{Definitions-Spherical Buildings and Lie Incidence Geometries}

\subsection{Coxeter systems and Coxeter complexes}

Definition 2.1 Let $W$ be a group generated by a finite nonempty set $S=\left\{s_{1}, \ldots, s_{n}\right\}$ of involutions and let, for each pair $\left(s_{i}, s_{j}\right) \in S \times S$, the number $m_{i j}$ be the order of the product $s_{i} s_{j}$ (setting $m_{i j}=\infty$ if $s_{i}, s_{j}$ generated an infinite group). Then $(W, S)$ is a Coxeter system if $W$ can be presented as $W=\left\langle S:\left(s_{i} s_{j}\right)^{m_{i j}}=1, \forall i, j \in\{1,2, \ldots, n\}\right\rangle$. The natural number $n$ is called the rank of the system.

The symmetric matrix $m_{i j}$ is called the Coxeter matrix belonging to $(W, S)$. The Coxeter diagram is the edge labelled graph $\Gamma(W, S)$ with vertex set $S$ and no edge between $s_{i}$ and $s_{j}$ if $m_{i j}=2$; otherwise an edge with label $\left(m_{i j}\right)$ between $s_{i}$ and $s_{j}$, for all $i, j \in\{1,2, \ldots, n\}$. The labels of edges with label (3) are usually omitted, those with label (4) are usually drawn as a a double edge, and those with label (6) are sometimes drawn as a triple edge. 
Let $(W, S)$ be a Coxeter system. If $S=S_{1} \cup S_{2}$, with $W=\left\langle S_{1}\right\rangle \times\left\langle S_{2}\right\rangle$ (then automatically $\left.S_{1} \cap S_{2}=\emptyset\right)$, then we say that $(W, S)$ is reduced. If $(W, S)$ is not reduced, then it is called irreducible.

In this paper we will only be concerned with finite irreducible Coxeter groups, that moreover arise as automorphism group of a crystallographic root system. We will not need the precise definition; it will suffice to know that crystallographic root systems are classified by Dynkin diagrams, which are Coxeter diagrams where every edge labelled $(\ell)$, with $\ell \geq 4$, gets an orientation.

Here is the list of Dynkin diagrams of irreducible crystallographic root systems (i.e., the corresponding Coxeter group is irreducible). The nodes of the diagram are labelled according to the standard conventions introduced by Bourbaki [1] (we can think of the node with label $i$ as the one corresponding with $s_{i} \in S$ ). Thinking away the arrow gives the corresponding Coxeter group.

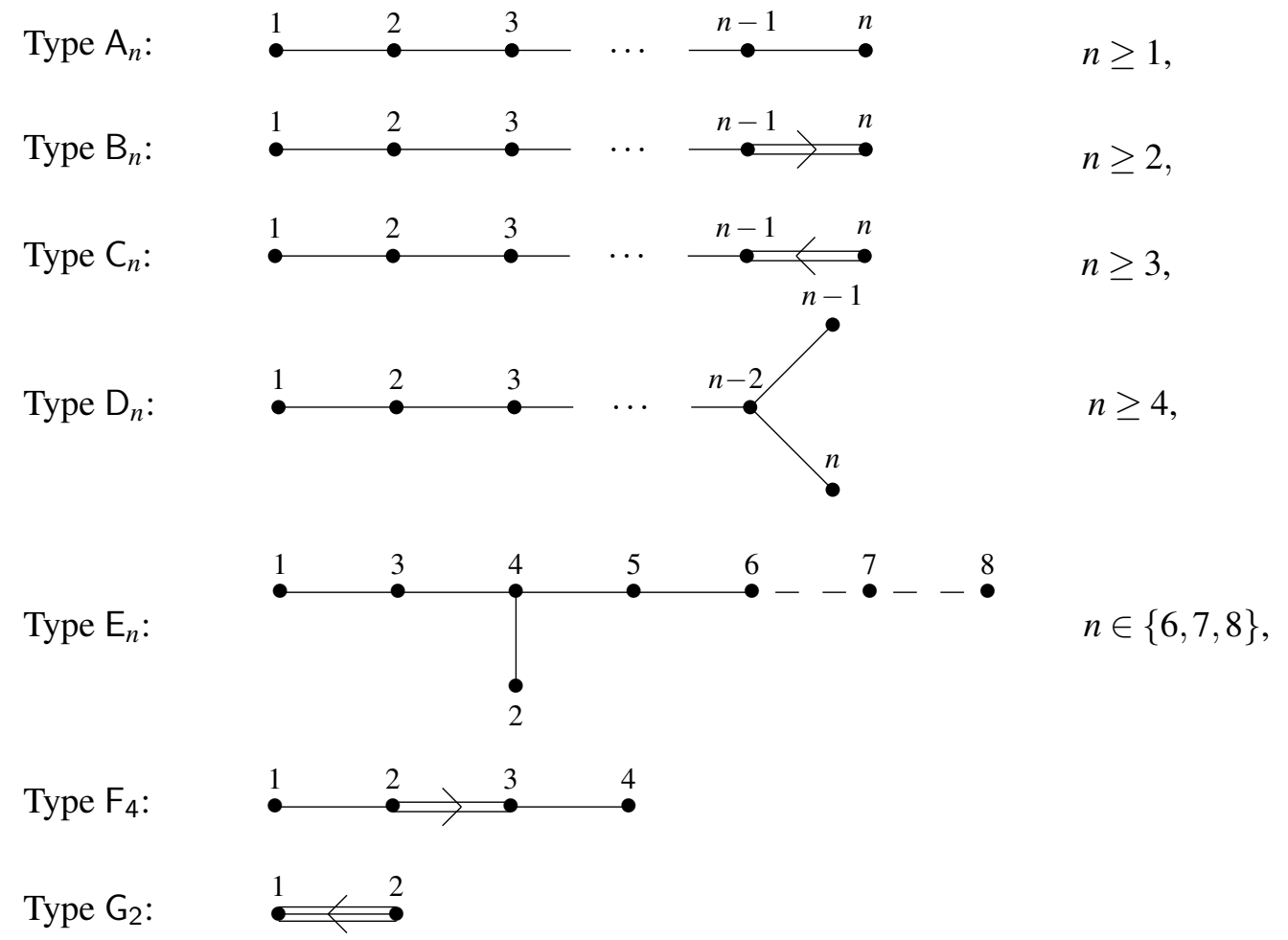

The irreducible finite Coxeter groups not arising from crystallographic root systems are the automorphism groups of the dodecahedron and the 600-cell in real Euclidean 4-space, and the finite dihedral groups $D_{2 n}$ (of which $D_{4}$ is reducible, $D_{6}$ is of type $A_{2}, D_{8}$ of types $\mathrm{B}_{2}$ and $\mathrm{C}_{2}$ and $D_{12}$ is of type $\mathrm{G}_{2}$ ).

The irreducible finite Coxeter groups and systems arising from the crystallographic root system of type $\mathrm{X}_{n}$, with $\mathrm{X} \in\{\mathrm{A}, \ldots, \mathrm{G}\}$ and $n$ appropriate, is called of type $\mathrm{X}_{n}$ itself. This is unambiguous except for types $B$ and $C$, which coincide for Coxeter groups and systems.

Definition 2.2 Let $(W, S)$ be a Coxeter system. A standard parabolic subgroup is a subgroup of $W$ generated by a proper subset of $S$. A parabolic subgroup is a conjugate of a standard parabolic subgroup. A maximal standard parabolic subgroup is one not contained in another one, i.e., generated by all but one elements of $S$. 
Definition 2.3 Recall that a simplicial complex consists of a set $X$, whose elements are usually called vertices, and a family $\Omega$ of subsets of $X$, called simplices, with the only condition that every subset of a member of $\Omega$ is also contained in $\Omega$. It is called a chamber complex if the maximal simplices, called chambers, are finite and all have the same cardinality, which is then called the rank of the complex. Two chambers $C$ and $C^{\prime}$ with $\left|C \backslash C^{\prime}\right|=1$ are called adjacent, and in this case $C \cap C^{\prime}$ is called a panel.

Definition 2.4 Let $(W, S)$ be a Coxeter system. Then we define a simplicial complex $\Delta(W, S)=$ $(X, \Omega)$ as follows. The set $X$ consists of the cosets of the maximal standard parabolic subgroups. A subset of $X$ belongs to $\Omega$ if it is the set of cosets of all maximal standard parabolic subgroups containing a coset of a not necessarily maximal standard parabolic subgroup.

It follows from the definition that $\Delta(W, S)$ is a chamber complex, that the chambers of $\Delta(W, S)$ are in one-to-one correspondence with the elements of $W$ and that chambers have size $|S|$. Moreover, every panel is contained in exactly two chambers, since the standard parabolic subgroup generated by a single element of $S$ has size 2 .

\subsection{Buildings and spherical buildings}

We are now ready to define the concept of a building.

Definition 2.5 Let $(W, S)$ be a Coxeter system. A building of type $(W, S)$ is a simplicial chamber complex $(X, \Omega)$ endowed with a family $\mathscr{A}$ of subcomplexes called apartments all isomorphic to $\Delta(W, S)$ such that

(B1) Every pair of simplices of $(X, \Omega)$ is contained in a member of $\mathscr{A}$;

(B2) If two simplices $A, A^{\prime}$ are contained in two apartments $\Sigma, \Sigma^{\prime}$, then there exists an isomorphism $\Sigma \rightarrow \Sigma^{\prime}$ fixing $A \cup A^{\prime}$ pointwise.

The Coxeter group $W$ is sometimes also called the Weyl group of the building. If $(W, S)$ is of type $\mathrm{X}_{n}$, with $\mathrm{X} \in\{\mathrm{A}, \ldots, \mathrm{G}\}$ and $n$ appropriate, then the building is also said to be of type $\mathrm{X}_{n}$ itself.

The definition of a building is rather abstract and does not immediately link up with incidence geometry. However, every building gives rise to many point-line incidence geometries by the following procedure.

Let $\Delta=(X, \Omega)$ be a building of type $(W, S)$. First we note that $\Delta$ is a numbered complex, i.e., one can assign types to the elements of $X$ such that every chamber contains exactly one vertex of each type. These types are in one-to-one correspondence with the elements of $S$. Indeed, if $C$ is a chamber and $x \in C$, then in any apartment containing $C$, the panel $C \backslash\{x\}$ corresponds to a coset of a standard parabolic subgroup of size 2, hence generated by a single element $s_{i} \in S$. Then we assign to $x$ the type $i$. The type $T$ of a simplex is then the union of the types of its elements, and the cotype is the complement $I \backslash T$. Set $I=\{1,2, \ldots, n\}$. Then we choose a subset $J \subseteq I$ and define the following point-line geometry $\Delta_{J}=\left(\mathscr{P}_{J}, \mathscr{L}_{J}\right)$. The set $\mathscr{P}_{J}$ of points is just the set of all simplices of type $J$. A generic line is the set of simplices of type $J$ for which the union with a fixed panel of cotype $j \in J$ is a chamber. We call $\Delta_{J}$ the $J$-Grassmannian geometry of $\Delta$.

In the present paper we are interested mainly in $J$-Grassmannian geometries of spherical buildings which arise from simple algebraic groups (a spherical building is a building with 
finite Weyl group). Given an isotropic algebraic group $G$ of relative rank $n$, there is a precise procedure that produces a spherical building of $\operatorname{rank} n, n \geq 2$. In fact, this procedure is exactly the same as above for Coxeter groups, if we choose a fixed Borel in $G$ and call the subgroups containing this Borel standard parabolic subgroups. When $G$ is defined over an algebraically closed field $\bar{k}$, then $G$ is completely determined by $\bar{k}$ and an irreducible crystallographic root system, or, equivalently, a connected Dynkin diagram $\mathrm{X}_{n}$. We will denote the corresponding building by $\mathrm{X}_{n}(\bar{k})$. Note that the corresponding Coxeter complex is also a building, and we denote it by $\mathrm{X}_{n}(1)$, alluding to the point of view that these Coxeter groups are algebraic groups over the field of order 1. If $k$ is finite, we also write $|k|$ instead of $k$.

Now, for an arbitrary field $k$, we can consider a simple algebraic group $G(\bar{k})$ over the algebraic closure $\bar{k}$ of $k$, and take the $k$-rational points (Galois descent) in such a way that the relative type (the corresponding Weyl group) is the same as the original one. In this case we denote the corresponding building by $\mathrm{X}_{n}(k)$. All such buildings are called split buildings.

For $J \subset I$, the $J$-Grassmannian geometry of $\mathrm{X}_{n}(k)$ is denoted by $\mathrm{X}_{n, J}(k)$ (where braces around the unique element of $J$ are omitted if $|J|=1$ ). These geometries are called (split) Lie incidence geometries. They are called simple when $|J|=1$, and they are called long root geometries if $J$ corresponds to the set of fundamental roots adjacent to the longest root. We have not defined the notions in the previous sentence, but it will suffice for us to list these subsets $J$ :

\begin{tabular}{l|c|c||l|c} 
type & $J$ & & type & $J$ \\
\hline $\mathrm{A}_{n}$ & $\{1, n\}$ & $n \geq 2$ & $\mathrm{E}_{6}$ & $\{2\}$ \\
\hline $\mathrm{B}_{n}$ & $\{2\}$ & $n \geq 2$ & $\mathrm{E}_{7}$ & $\{1\}$ \\
\hline $\mathrm{C}_{n}$ & $\{1\}$ & $n \geq 3$ & $\mathrm{E}_{8}$ & $\{8\}$ \\
\hline $\mathrm{D}_{n}$ & $\{2\}$ & $n \geq 4$ & $\mathrm{~F}_{4}$ & $\{1\}$ \\
\hline & & & $\mathrm{G}_{2}$ & $\{2\}$
\end{tabular}

Now given a finite Coxeter system $(W, S)$, there are other buildings of type $(W, S)$ than the ones listed above. Tits [7] proves that, if the rank is at least 3, then every spherical building arises from an algebraic group in the broad sense (including classical groups and groups of mixed type). We will not introduce notations for all cases, but we content ourselves with mentioning the following terminology. We use the labelling of the diagrams introduced above.

1. If $\Delta$ is a building of type $\mathrm{A}_{n}$, then the 1-Grassmannian is the point-line truncation (i.e., the restriction to the points and the lines) of a projective space. If the projective space arises from a vector space over a commutative field, then this 1-Grassmannian is the Lie incidence geometry $\mathrm{A}_{n, 1}(k)$. If the projective space arises from a vector space over a non-commutative skew field $\ell$, then we extend the previous notation substituting $k$ with $\ell$. If $n=2$, there are other cases which we do not need.

2. If $\Delta$ is a building of type $\mathrm{B}_{n}$ (or equivalently, of type $\mathrm{C}_{n}$ if $n \geq 3$; for $n=2$ the Bourbaki labelling does not agree), then the 1-Grassmannian is a polar space.

(a) If this polar space arises from a parabolic quadric in $\mathrm{PG}(2 n, k)$, for some field $k$ and $n \geq 2$, i.e., from a non-degenerate symmetric bilinear form of Witt index $n$ in a $(2 n+1)$-dimensional vector space, then $\Delta$ coincides with $\mathrm{B}_{n}(k)$. 
(b) If this polar space arises from a linear line complex in $\mathrm{PG}(2 n-1, k)$, for some field $k$ and $n \geq 3$, i.e., from a non-degenerate alternating bilinear form in a $2 n$ dimensional vector space, then $\Delta$ coincides with $C_{n}(k)$.

(c) If this polar space arises from a hyperbolic quadric in $\mathrm{PG}(2 n-1, k)$, for some field $k$ and $n \geq 4$, i.e., from a non-degenerate symmetric bilinear form of Witt index $n$ in a $2 n$-dimensional vector space, then $\Delta$ coincides with $\mathrm{D}_{n}(k)$.

For the specific case $n=2$, see below. Notice that also type $\mathrm{D}_{n}$ gives rise to polar spaces and to buildings of type $\mathrm{B}_{n}$. Indeed, the so-called flag complex of a hyperbolic quadric in $\mathrm{PG}(2 n-1, k)$ is a building of type $\mathrm{B}_{n}$, but the so-called oriflamme complex is a building of type $\mathrm{D}_{n}$. The difference between the two is that the latter is a thick building, meaning that every panel is contained in at least three chambers, while the former is not. There is a procedure to produce a thick building from every non-thick spherical one, except in the thin case, i.e., when every panel is contained in exactly two chambers, and then we have a Coxeter complex. So usually, one is only interested in thick spherical buildings.

3. All thick buildings of types $\mathrm{D}_{n}, n \geq 4$ and $\mathrm{E}_{m}, m \in\{6,7,8\}$, are isomorphic to $\mathrm{D}_{n}(k)$, $\mathrm{E}_{m}(k)$, respectively, for some commutative field $k$.

4. The 1- or 4-Grassmannian of any thick building of type $\mathrm{F}_{4}$ is called a metasymplectic space.

In general, the $J$-Grassmannian geometry $\Delta_{J}$, for a spherical building $\Delta$, will be called a Lie incidence geometry, and it is called simple if $|J|=1$.

Let $\Delta$ be a building of type $(W, S)$. Then the Coxeter diagram of $(W, S)$ can be used to deduce the structure of the links. More precisely, let $F$ be a simplex of $\Delta$ with $|F| \leq|S|-2$, then we can consider the set $\Delta_{F}$ of all simplices $F^{\prime}$ of $\Delta$ disjoint from $F$ such that $F \cup F^{\prime}$ is a simplex. This forms a simplicial chamber complex again, called the residue in $\Delta$ of $F$, and it is a building of type $\left(W^{\prime}, S^{\prime}\right)$, where $S^{\prime}$ corresponds to the cotype of $F$, and $W^{\prime}$ is generated by $S^{\prime}$. Hence, in order to have a geometric feeling for buildings, we can do it inductively and start with rank 2, i.e., $|S|=2$. In this case, the simplicial complex consists of singletons and pairs; the singletons are numbered 1 or 2 and every pair contains a vertex of either type. Hence we obtain a bipartite graph. In the non-spherical case, a bipartite graph corresponds to a building if and only if the graph is a (necessarily infinite) tree without vertices of valency 1 . In the spherical case, the axioms (B1) and (B2) translate into the following definition.

Let $\Gamma=(V, E)$ be a bipartite graph. Then $\Gamma$ is called a generalized $n$-gon if the following conditions hold.

(GP1) The diameter of $\Gamma$ is $n$;

(GP2) The girth of $\Gamma$ is $2 n$.

If we restrict to thick buildings, then we restrict to bipartite graphs with bivalency $(s, t)$, where $s, t \geq 3$. If $n$ is odd, however, then $s=t$. This has the following consequence for a general thick building. Let $\Delta$ be a thick building of type $(W, S)$. Delete in the Coxeter graph all edges labelled $(\infty)$ and delete the isolated vertices of the graph obtained. Then delete all edges with even label. Then every connected component $\Gamma^{*}$ of the resulting graph can be 
assigned a cardinal number $t$ such that, if $s_{i} \in S$ corresponds with any vertex of $\Gamma^{*}$, then the number of chambers containing any panel of cotype $s_{i}$ is equal to $t+1$. In the irreducible spherical case, this means that with every building $\Delta$ of type $\mathrm{A}_{n}, \mathrm{D}_{n}$ and $\mathrm{E}_{n}$ is associated a unique cardinal number $t \geq 2$ such that every panel is contained in exactly $t+1$ chambers. We say that $t$ is the order of $\Delta$. For the other types, there are two such cardinal numbers $s, t$, and we say that $\{s, t\}$ is the order. In the case where the rank is at least 3 and the building is finite, the orders are always powers of a prime number.

Example 2.6 Let $\Gamma$ be a thick generalized 3-gon. Then $\Gamma$ is the incidence graph of a projective plane. Hence the notions of a thick building of type $A_{2}$ and a projective plane are equivalent.

Example 2.7 Let $\Gamma$ be a generalized 4-gon. Then $\Gamma$ is the incidence graph of a generalized quadrangle, i.e., a point-line incidence structure such that two points determine at most one line, no point is contained in all lines, no line contains all points, and for each point $p$ and each line $L$ not incident with $p$, there exists a unique point $q$ incident with $L$ and a unique line $M$ incident with $p$ such that $q$ is incident with $M$.

We now present two specific examples of generalized quadrangles. The first one is the building $B_{2}(2)$ and the second one is not related to a Dynkin type.

Example 2.8 In general, the Lie incidence geometry $\mathrm{B}_{2,2}(k)$ is the generalized quadrangle, also denoted by $W(k)$, arising from a symplectic polarity or linear line system in $\mathrm{PG}(3, k)$ (or a non-degenerate alternating form in a 4-dimensional vector space over $k$ ). If $k$ is perfect and has characteristic 2, then the Lie incidence geometries $\mathrm{B}_{2,2}(k)$ and $\mathrm{B}_{2,1}(k)$ are isomorphic. This happens for instance for $|k|=2$. In this case, there is a combinatorial description of this geometry $W(2)$, or of the corresponding building, or of the corresponding bipartite graph (the incidence graph). Indeed, one considers a 6-set $N=\{1,2,3,4,5,6\}$. The vertices of one bipartition class are the pairs of $N$, and the vertices of the other bipartition class are the partitions of $N$ into 2-sets. Adjacency is natural (based on inclusion). We will use this representation below. Note that the order of $W(2)$, as a building, is $\{2,2\}$.

We now introduce the unique generalized quadrangle $Q(2,4)$ with lines of size 3 and such that every point is incident with exactly 5 lines (hence it has order $\{2,4\}$ ).

Example 2.9 Let $N=\{1,2,3,4,5,6\}$ and $N^{\prime}=\left\{1^{\prime}, 2^{\prime}, 3^{\prime}, 4^{\prime}, 5^{\prime}, 6^{\prime}\right\}$. Then we define the point set of $Q(2,4)$ as $N \cup N^{\prime} \cup\left(\begin{array}{c}N \\ 2\end{array}\right)$, where $\left(\begin{array}{c}N \\ 2\end{array}\right)$ is the set of all 2-subsets of $N$. The lines are given by the partitions of $N$ into 2-sets (as above for $W(2)$ ), and also by the triples $\left\{i,\{i, j\}, j^{\prime}\right\}$, for every ordered pair $(i, j) \in N \times N$ with $i \neq j$.

\subsection{Graphs, embeddings and more conventions and notation}

2.3.1 Graphs Given a spherical building $\Delta$, there are a lot of graphs that one can associate with it. We will be mostly interested in incidence graphs and collinearity graphs. The full incidence graph of a building is just the 1-skeleton of $\Delta$ as a simplicial complex, i.e., only considering the vertices and the simplices of size 2 . For buildings of rank 2, this is common to use. One can also restrict to certain types, but more common is to use the incidence graph of Lie incidence geometries related to $\Delta$, i.e., the vertices of the graph are the 
points and lines of a certain Lie incidence geometry, and adjacency in the graph is incidence in the geometry.

For Lie incidence geometries, another commonly used graph is the collinearity graph whose vertex set is just the points set of the geometry, two distinct vertices being adjacent if the corresponding points are collinear (are contained in a common line).

2.3.2 Embeddings An embedding of a Lie incidence geometry $(\mathscr{P}, \mathscr{L})$ into a projective space $\mathrm{PG}(d, k)$ consists of an injective mapping of $\mathscr{P}$ into the point set of PG $(d, k)$ such that all points of any line $L \in \mathscr{L}$ are mapped onto all points of a line of PG $(d, k)$. The theory of representations of algebraic groups yields embeddings of many Lie incidence geometries. We will construct some of those in this paper in an alternative way.

To accomplish this we will have to use some notions typical for projective geometry.

Let PG $(d, k)$ be the $d$-dimensional projective space over the commutative field $k$. Then we can assign to a quadruple $\left(p_{1}, p_{2} ; q_{1}, q_{2}\right)$ of collinear points a unique scalar $r \in k$, called the cross-ratio. It is a number invariant under any linear permutation and base-change.

Dually, the cross-ratio can also be defined for a quadruple of concurrent hyperplanes. It can be defined directly by considering a line disjoint from the intersection of the hyperplanes and then identifying the hyperplanes with their intersections with this line. Likewise, one can define in a completely similar way the cross ratio of every quadruple of concurrent $i$ subspaces, i.e., four $i$-spaces having an $(i-1)$-space in common and being contained in a common $(i+1)$-space.

An abstract Segre geometry $\mathscr{S}_{n, m}(k)$ is a Lie incidence geometry $\mathrm{A}_{n, 1}(k) \times \mathrm{A}_{m, 1}(k)$. It has a canonical embedding in the projective space $\mathrm{PG}(n m+n+m, k)$. Indeed, we can map the point $\left(\left(x_{i}\right)_{0 \leq i \leq n},\left(y_{j}\right)_{0 \leq j \leq m}\right)$ to the point $\left(x_{i} y_{j}\right)_{0 \leq i \leq n ; 0 \leq j \leq m}$. This canonical embedding is called a Segre variety. There are many geometric constructions; we mention one below in Section 5.

When $n=1$, then fix a maximal 1-space $L$. Every $m$-space of $\mathscr{S}_{1, m}(k)$ (if $m=1$, then we refer to a 1-space of the other system) intersects $L$ in a unique point, and hence this induces a cross-ratio for the $m$-spaces. It is independent of the choice of $L$ (but it is dependent on the embedding, hence it is only defined for Segre varieties, and not for arbitrary Segre geometries).

Finally, for an arbitrary point of an arbitrary point-line geometry, we denote collinearity always by $\perp$; in particular $x^{\perp}$ always means the set of points collinear to the point $x$.

\section{Intersections of Quadrics}

In this section we will describe a method to construct the smallest dimensional representations of the buildings of type $\mathrm{E}_{6}$ and $\mathrm{E}_{7}$ over a field $k$, namely those in respective projective dimensions 26 and 55, as an intersection of (degenerate) quadrics. For type $E_{6}$, such a set of quadrics was given by Cohen [2], but we present a combinatorial logical way to write these down, as opposed to just list them.

\subsection{A baby example}

It is well know that there is a very explicit way to write any Grassmannian of any projective space as a subset of quadrics, see Hirschfeld \& Thas [4]. We will present the 
example of the line Grassmannian $A_{5,2}(k)$ of a projective 5-space over any field $k$ (which corresponds to the third cell in the second row of the Freudenthal-Tits Magic Square).

A generic point of $A_{5,2}(k)$ has coordinates of the form

$$
\left(p_{i j}\right)_{1 \leq i<j \leq 6}, \text { where } p_{i j}=\left|\begin{array}{cc}
x_{i} & x_{j} \\
y_{i} & y_{j}
\end{array}\right|,
$$

for two independent vectors $\left(x_{i}\right)_{1 \leq i \leq 6}$ and $\left(y_{i}\right)_{1 \leq i \leq 6}$. If we set $p_{j i}=-p_{i j}$, then $A_{5,2}(k)$ is contained in the quadric with equation $p_{i j} p_{m \ell}+p_{i \ell} p_{j m}+p_{i m} p_{\ell j}=0$, with $i, j, m, \ell \in$ $\{1,2, \ldots, 6\}$ all distinct. Varying over all possibilities, the intersection of all such quadrics yields exactly $A_{5,2}(k)$. In fact, it is easy to see that we can restrict to all $i, j, m, \ell$ with $1 \leq i<j<m<\ell \leq 6$. In this case, we even do not need to define $p_{j i}$ for $j>i$, since we can write the above equation as $p_{i j} p_{m \ell}+p_{i \ell} p_{j m}=p_{i m} p_{j \ell}$. So $A_{5,2}(k)$ is the intersection of 15 quadrics. Note that it lives in 14-dimensional space, so the vector dimension of the embedding is equal to the number of quadrics. A similar thing will happen for the Lie incidence geometry $\mathrm{E}_{6,1}(k)$ of exceptional type.

\subsection{Buildings of type $E_{6}$}

The combinatorial data underlying the subscripts of the coordinates of our baby example were the 2-subsets of a 6-set (the 2-subsets can be taken as unordered by insisting that $p_{i j}$ is only defined for $i<j$ ). It is well known that the 2-subsets of a 6 -set can be identified with the points of the smallest nontrivial generalized quadrangle denoted by $W(2)$, where the lines are the partitions of the 6-set into three 2-subsets. The subscripts of the coordinates appearing in the equation of a single quadric then correspond with the points collinear to a fixed point of $W(2)$ (and there are 15 points in $W(2)$, so all points appear as this fixed point). Within the equation of a fixed quadric, the subscripts of the coordinates that are multiplied together, correspond to collinear points, while those that are in distinct terms are always non-collinear. However, there is no homogeneous description of the subscripts of the coordinates of the term that has to go into the right hand side.

We generalize the above construction as follows. Let $Q(2,4)$ be the unique generalized quadrangle of order $(2,4)$ with points set $\mathscr{P}$ and line set $\mathscr{L}$. As already mentioned before, we have $|\mathscr{P}|=27$ and $|\mathscr{L}|=45$. Every line is incident with three points and every point is collinear with 10 other points. Let a basis of $\mathrm{PG}(26, k)$ be indexed by the points of $Q(2,4)$. Hence an arbitrary point has coordinates of the form $\left(x_{i}\right)_{i \in \mathscr{P}}, x_{i} \in k$ for all $i \in \mathscr{P}$. Given a point $i \in \mathscr{P}$ and a line $L \in \mathscr{L}$ with $i \in L$, we define the quadric $Q_{i, L}$ with equation

$$
x_{j_{1}} x_{j_{2}}+x_{j_{3}} x_{j_{4}}+x_{j_{5}} x_{j_{6}}+x_{j_{7}} x_{j_{8}}=x_{j_{9}} x_{j_{10}},
$$

where $i^{\perp}=\left\{j_{k}: k=1,2, \ldots, 10\right\}$ and $L=\left\{i, j_{9}, j_{10}\right\}$. If we want a set of 27 quadrics, like in Cohen, or similarly as in the baby example above, then we should make a unique choice for $L$ given $i$. This is accomplished by introducing a spread. A spread of $Q(2,4)$ is a partition of $\mathscr{P}$ into nine lines. It turns out that, up to isomorphism, $Q(2,4)$ contains exactly two spreads. Only one is a so-called regular spread $\mathscr{S}$, i.e., given any pair of lines $L_{1}, L_{2} \in \mathscr{S}$, the unique line $L_{3}$ composed of the three points outside $L_{1} \cup L_{2}$ that are collinear with collinear points of $L_{1} \cup L_{2}$ also belongs to $\mathscr{S}$. The lines $L_{1}, L_{2}, L_{3}$ form a regulus, i.e., a set of three lines such that every line of $Q(2,4)$ intersecting two of them also intersects the third. The reguli

of $\mathscr{S}$ define an affine plane of order 3 on $\mathscr{S}$.

Now we have the following result. 
Theorem 3.1 The Lie incidence geometry $\mathrm{E}_{6,1}(k)$ is the intersection of the twenty seven quadrics $Q_{i, L}$ in $\mathrm{PG}(26, k)$, with $i$ ranging over $\mathscr{P}$ and $L$ the unique member of $\mathscr{S}$ incident with $i$.

Preparing for the construction of the Lie incidence geometry $E_{7,7}(k)$ in the next subsection, we make a few additional observations regarding the case $\mathrm{E}_{6,1}(k)$.

Consider the complement $\Gamma^{e_{6}}$ of the collinearity graph of $Q(2,4)$. It is well known that this is isomorphic to the thin Lie incidence geometry $\mathrm{E}_{6,1}(1)$. We identify each vertex of $\Gamma^{e_{6}}$ with the corresponding basis point of PG $(26, k)$. Then every vertex of $\Gamma^{e_{6}}$ belongs to $Q_{i, L}$, for all $i \in \mathscr{P}$ and $L \ni i$ in $\mathscr{S}$. Moreover, two basis points $i, j$ are non-adjacent as vertices of $\Gamma^{e_{6}}$ if and only if they are opposite points of precisely one of those quadrics, namely $Q_{k, M}$, with $\{i, j, k\}$ a line of $Q(2,4)$ and $k \in M \in \mathscr{S}$. In this case, $i$ and $j$ are incident with a unique element $\Sigma$ of type 6 of $\mathrm{E}_{6,1}(1)$, which is isomorphic to the Lie incidence geometry $\mathrm{D}_{5,1}(1)$. The points of $\Sigma$ are precisely the subscripts appearing in the equation of $Q_{k, M}$.

\subsection{Buildings of type $E_{7}$}

We now construct the Lie incidence geometry $E_{7,7}(k)$ as an intersection of quadrics. The Lie incidence geometries of the last three subsections, $\mathrm{A}_{5,2}(k), \mathrm{E}_{6,1}(k), \mathrm{E}_{7,7}(k)$ are situated as follows in the Freudenthal-Tits Magic Square (and we only write the types):

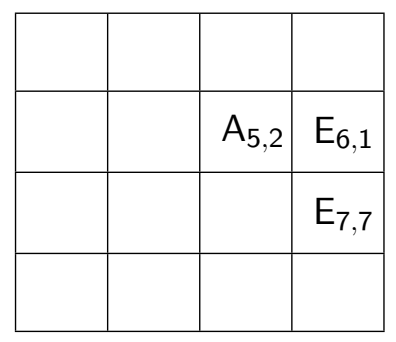

Hence just as $A_{5,2}(k)$ served as a baby example for $E_{6,1}(k)$ because it is at the left of it, $\mathrm{E}_{6,1}(k)$ serves as a warming-up for $\mathrm{E}_{7,7}(k)$ because it is just above it.

It is well known that the Gosset graph is a model for the thin Lie incidence geometry $E_{7,7}(1)$. Let us therefore denote the Gosset graph by $\Gamma^{e_{7}}$. Vertices of type 1 of $E_{7}(1)$ correspond to copies of $D_{6,1}$. There are 126 such copies inside $\Gamma^{e_{7}}$. Also, $\Gamma^{e_{7}}$ has 56 vertices. We again identify the vertices of $\Gamma^{e_{7}}$ with the points of a basis of $\operatorname{PG}(55, k)$. It turns out, similarly as above for $\mathrm{E}_{6,1}(k)$, that the representation of the Lie incidence geometry $\mathrm{E}_{7,7}(k)$ in $\operatorname{PG}(55, k)$ is contained in the intersection of 126 quadrics $Q_{\Sigma}$ with equations of the form (signs being indetermined for now, hence the \pm notation)

$$
\sum_{a=1}^{6} \pm x_{i_{2 a-1}} x_{i_{2 a}}=0
$$

where $\left\{i_{1}, i_{2}, \ldots, i_{12}\right\}$ is the set of points of a copy $\Sigma$ of $\mathrm{D}_{6,1}$ inside $\Gamma^{e_{7}}$. We first determine the signs in these equations. This is not possible by one homogeneous rule, i.e., one cannot choose the signs in such a way that all equations have the same number of plus and minus signs. Instead there are two different possiblities. In order to explain this, we need the following construction of the Gosset graph.

Consider two copies $\Gamma_{1}=\left(V_{1}, E_{1}\right)$ and $\Gamma_{2}=\left(V_{2}, E_{2}\right)$ of $\Gamma^{e_{6}}$, and let $\theta$ be an isomorphism between them (we shall use $\theta$ in both directions, i.e., we view $\theta$ as an involutory 
permutation of $V_{1} \cup V_{2}$ interchanging $\Gamma_{1}$ and $\Gamma_{2}$ ). Let $\infty_{1}, \infty_{2}$ be two symbols not belonging to $V_{1} \cup V_{2}$. Then $\Gamma^{e_{7}}=(V, E)$ can be described as follows. The vertex set $V$ is equal to $\left\{\infty_{1}\right\} \cup V_{1} \cup V_{2} \cup\left\{\infty_{2}\right\}$. Adjacency inside $V_{1}$ and $V_{2}$ is the one of $\Gamma_{1}$ and $\Gamma_{2}$, respectively. The vertex $\infty_{a}$ is adjacent to all vertices in $V_{a}, a=1,2$. Finally, a vertex $v_{1} \in V_{1}$ is adjacent to a vertex $v_{2} \in V_{2}$ if $v_{1}^{\theta}$ is not adjacent to $v_{2}$ in $\Gamma_{2}$ (which is equivalent to $v_{1}$ not being adjacent to $v_{2}^{\theta}$ in $\Gamma_{1}$ ).

Note that, in $\Gamma^{e_{6}}$, the graph induced on the set of vertices not adjacent to a given vertex is precisely a copy of $D_{5,1}(1)$. Hence every vertex in $V_{1}$ or $V_{2}$ is adjacent to a set of vertices of $V_{2}$ or $V_{1}$, respectively, for which the induced subgraph is $D_{5,1}(1)$. Let us briefly call a copy of $D_{5,1}(1)$ and $D_{6,1}(1)$ in $\Gamma^{e_{6}}$ and $\Gamma^{e_{7}}$, respectively, a $D_{5}$ and a $D_{6}$, respectively. Let $\{a, b\}=\{1,2\}$. Pick $v_{a} \in V_{a}$ arbitrary. Then the set $W_{a}$ of points in $V_{b}$ collinear to $v_{a}$ is a $D_{5}$ in $\Gamma_{b}$; hence it is easy to see that $W_{a} \cup\left\{v_{a}, \infty_{b}\right\}$ is a $D_{6}$ in $\Gamma^{e_{7}}$. This way, we recover $2 \times 27=54$ subgraphs $D_{6}$ of the 126 in total in $\Gamma^{e 7}$. We call these of type 1 .

Now let $P$ be a vertex of type 2 in the building $\mathrm{E}_{6}(1)$. In the Lie incidence geometry $\mathrm{E}_{6,1}(1), P$ corresponds to the subgraph isomorphic to the Lie incidence geometry $A_{5,1}(1)$. This, in turn, corresponds to a 6-clique in the graph $\Gamma^{e_{6}}$. So let $P$ be a 6-clique in, say, $\Gamma_{1}$. There is a unique 6-clique $P^{\prime}$ in $\Gamma_{1}$ (the opposite one) with the property that $P^{\prime}$ is incident with each $D_{5}$ opposite some element of $P$. This can best be seen in $Q(2,4)$ as follows. The set $P$ is a set of six points no two of which are collinear. Let $i_{1}$ be one of them. Then there exists a point $j_{1}$ of $Q(2,4)$ opposite $i_{1}$ such that $P$ is equal to $\left\{i_{1}\right\}$ union the set of points of $Q(2,4)$ collinear with $j_{1}$ but not collinear with $i_{1}$. Then $P^{\prime}$ is the union of $\left\{j_{1}\right\}$ with the set of points of $Q(2,4)$ collinear with $j_{1}$ but not collinear with $i_{1}$. Clearly, each point of $P$ is not collinear to a unique point of $P^{\prime}$ and vice versa. This means that, in $\Gamma_{1}$, each element of $P$ is adjacent to a unique element of $P^{\prime}$. Hence each element of $P^{\prime}$ is not adjacent to a unique element of $P^{\theta}$. Consequently $P^{\prime} \cup P^{\theta}$ is a $D_{6}$ in $\Gamma^{e_{7}}$, called of type 2 . Since there are $72=126-54$ vertices of type 2 in $E_{6}(1)$, this takes care of the other subgraphs $D_{6}$ in $\Gamma^{e_{7}}$.

We can now define the signs in the equation of $Q_{\Sigma}$, for each $D_{6}$. So let $\Sigma$ be any $D_{6}$. We fix a regular spread $\mathscr{S}_{1}$ in the generalized quadrangle corresponding to $\Gamma_{1}$ and we let $\mathscr{S}_{2}$ be its image under $\theta$. First let $\Sigma$ be of type 1, and suppose, to fix the ideas, that $\Sigma$ contains $\infty_{1}$. Then exactly two non-collinear vertices $i$ and $j$ of $\Sigma \cap V_{1}$ belong to a common member of $\mathscr{S}_{1}$ and we define all the signs in the equation of $Q_{\Sigma}$ to be positive, except for the sign of the term $x_{i} x_{j}$, which is defined to be negative.

Now let $\Sigma$ be of type 2 and suppose $\Sigma=P_{1} \cup P_{2}$, with $P_{a}$ a 6-clique in $\Gamma_{a}, a=1,2$. We define the following set $\Pi$ of thin projective planes in $\Gamma_{1}$. The set $\Pi$ will have the property that each line of $\Gamma_{1}$ is incident with a unique member of $\Pi$. Let $L$ be a thin line of $\Gamma_{1}$, i.e., an edge $\{i, j\}$. Then $i$ and $j$ can be seen as two non-collinear points of $Q(2,4)$. Let $L, M$ be the members of $\mathscr{S}$ containing $i, j$, respectively. Let $\{L, M, N\}$ be the regulus of $Q(2,4)$ containing $L$ and $M$. Then $N \in \mathscr{S}$ and there is a unique point $\ell$ on $N$ collinear in $Q(2,4)$ to neither $i$ nor $j$. Hence $\{i, j, \ell\}$ is a thin projective plane in $\Gamma_{1}$. We would have obtained the same plane starting from the edges $\{i, \ell\}$ and $\{j, \ell\}$. Hence each line of $\Gamma_{1}$ is incident with a unique member of $\Pi$. A double count now reveals that $|\Pi|=72$.

Below we show that each 6-clique of $\Gamma_{1}$ contains exactly two members of $\Pi$, which moreover are disjoint. Let $\pi$ and $\pi^{\prime}$ be the two members of $\Pi$ contained in $P_{1}$. Then we give the terms of the equation of $Q_{\Sigma}$ containing subscripts in different planes $\pi$ and $\pi^{\prime}$ different signs. This completes the description of 126 quadrics which all contain $\mathrm{E}_{7,7}(k)$.

We still need three quadrics to completely determine $\mathrm{E}_{7,7}(k)$. Indeed, opposite points in $\Gamma^{e^{7}}$ do not appear as subscripts in a common equation yet, hence the corresponding points 
are collinear in the intersection of the 126 quadrics, whereas these points are not collinear in $\mathrm{E}_{7,7}(k)$ (neither in its embedding in $\mathrm{PG}(55, k)$ ).

We construct a set of 63 quadrics $Q_{\Sigma, \Sigma^{\prime}}$ with equation

$$
\sum_{a=1}^{12} \pm x_{i_{a}} x_{i_{a+12}}=0
$$

where $\left\{i_{1}, i_{2}, \ldots, i_{12}\right\}$ is the set of points of a copy $\Sigma$ of $\mathrm{D}_{6,1}(1)$ inside $\Gamma^{e_{7}}$, and $\left\{i_{13}, i_{14}, \ldots, i_{24}\right\}$ is the unique other copy $\Sigma^{\prime}$ of $D_{6,1}(1)$ opposite $\Sigma$, i.e., every point $i_{j}$ of $\Sigma$ is opposite (at distance 3 of) a unique point $i_{j+12}$ of $\Sigma^{\prime}$ (so $\Sigma^{\prime}$ just consists of the opposites of $\Sigma$ ), $1 \leq j \leq 12$. There are two possibilities.

1. Suppose first that $\infty_{1} \in \Sigma$. Then $\infty_{2} \in \Sigma^{\prime}$. Let $i_{1}$ and $i_{2}$ be the vertices of $\Sigma$ and $\Sigma^{\prime}$, respectively, opposite $\infty_{1}$ and $\infty_{2}$, respectively, in $\Sigma$ and $\Sigma^{\prime}$, respectively. Then the two terms $X_{\infty_{1}} X_{\infty_{2}}$ and $X_{i_{1}} X_{i_{2}}$ get the same sign, and all the others the opposite sign. Note there are 27 such quadrics.

2. If $\infty_{1} \notin \Sigma \cup \Sigma^{\prime}$, then we may choose the indices such that $\left\{i_{1}, \ldots, i_{6}\right\}$ and $\left\{i_{7}, \ldots, i_{12}\right\}$ are 6-cliques of $\Gamma^{e_{7}}$ contained in $\Gamma_{1}$. Then $\left\{i_{13}, \ldots, i_{18}\right\}$ and $\left\{i_{19}, \ldots, i_{24}\right\}$ are two 6cliques in $\Gamma_{2}$. We then choose the signs of $X_{i_{1}} X_{i_{13}}, \ldots, X_{i_{6}} X_{i_{18}}$ all equal, and those of $X_{i_{7}} X_{i_{19}}, \ldots, X_{i_{12}} X_{i_{24}}$ get the opposite sign. Note that there are 36 such quadrics.

We now have the following theorem.

Theorem 3.2 (i) The intersection of the 126 quadrics $Q_{\Sigma}$, with $\Sigma$ a subgraph of $\Gamma^{e_{7}}$ isomorphic to $\mathrm{D}_{6,1}(1)$, and the 63 quadrics $Q_{\Sigma, \Sigma^{\prime}}$, with $\Sigma$ and $\Sigma^{\prime}$ opposite copies in $\Gamma^{e_{7}}$ isomorphic to $\mathrm{D}_{6,1}(1)$, is an embedding of the Lie incidence geometry $\mathrm{E}_{7,7}(k)$ in PG $(55, k)$.

(ii) Let $i_{1}, i_{2}, i_{3}$ be three vertices of $\Gamma_{2}$ corresponding to the points on a line of the generalized quadrangle $Q(2,4)$ underlying $\Gamma_{2}$ (said differently, $\left\{i_{1}, i_{2}, i_{3}\right\}$ is a maximal coclique of $\left.\Gamma_{2}\right)$. Then the intersection of the 126 quadrics $Q_{\Sigma}$, with $\Sigma$ a subgraph of $\Gamma^{e_{7}}$ isomorphic to $\mathrm{D}_{6,1}(1)$, and the 3 quadrics $Q_{\Sigma, \Sigma^{\prime}}$, with $\Sigma$ and $\Sigma^{\prime}$ opposite copies in $\Gamma^{e_{7}}$ isomorphic to $\mathrm{D}_{6,1}(1)$, with $\infty_{1} \in \Sigma$ and $\left\{i_{1}, i_{2}, i_{3}\right\} \cap \Sigma \neq \emptyset$, is an embedding of the Lie incidence geometry $\mathrm{E}_{7,7}(k)$ in $\mathrm{PG}(55, k)$.

We finally prove the result announced and used above.

Proposition 3.3 Let $\mathscr{S}$ be a regular spread of $Q(2,4)$ and let $\Pi$ be a set of 72 thin projective planes constructed as above such that every thin line of $\mathrm{E}_{6,1}(1)$ is contained in precisely one member of $\Pi$. Then every thin 5-space of $\mathrm{E}_{6,1}(1)$ contains exactly two members of $\Pi$, which moreover are disjoint.

Proof Let $P$ be a 5-space of $\mathrm{E}_{6,1}(1)$, hence a 6 -clique in $\Gamma^{e_{6}}$. For each point $i_{1} \in P$, we now construct a member of $\Pi$ containing $i_{1}$ and contained in $P$. Indeed, as above, there exists a point $j_{1}$ of $Q(2,4)$ opposite $i_{1}$ such that $P$ is equal to $\left\{i_{1}\right\}$ union the set $\left\{i_{2}, i_{3}, i_{4}, i_{5}, i_{6}\right\}$ of points of $Q(2,4)$ collinear with $j_{1}$ but not collinear with $i_{1}$. Let $L_{1}$ be the unique member of $\mathscr{S}$ containing $i_{1}$. We can choose the indices so that the line of $Q(2,4)$ joining $j_{1}$ with $i_{2}$ meets $L$. Let $M$ be the unique member of $\mathscr{S}$ containing $j_{1}$. Then we can choose indices such that $i_{3} \in M$. Then clearly $\left\{i_{1}, i_{2}, i_{3}\right\} \in \Pi$. 
Considering $i_{4}$, we can construct a second member of $\Pi$ in $P$. Hence every thin 5-space contains at least two members of $\Pi$. A double count of the pairs $\left(P^{\prime}, \pi\right)$, with $P^{\prime}$ a 5 -space of $\mathrm{E}_{6,1}(1)$ and $\pi \in \Pi$ with $\pi \subseteq P^{\prime}$, reveals that this must be exactly two. Since every point of $P$ must be contained in a member of $\Pi$ contained in $P$, the two members of $\Pi$ in $P$ must cover all points and hence are disjoint.

\section{Equator and Trace Geometries}

In this section, we construct Lie incidence geometries of lower rank inside Lie incidence geometries of higher rank. This might seem like a trivial exercise from the point of view of buildings, thinking about residues, but it is actually an interesting general open question which Lie incidence geometries are contained in a given one. The constructions that we will present give ordinary and predictable residue geometries, but also a geometric interpretation of inclusions of quadratic composition algebras. Hence it happens typically for the geometries of the Freudenthal-Tits Magic Square, since the construction of the Lie algebra in the cell $(i, j)$ uses quadratic alternative composition algebras of dimensions $2^{i-1}$ and $2^{j-1}$ over the base field.

Also, we will use equator geometries in the next two sections, where they will prove a useful tool.

All in all, we will treat the following cells in the Freudenthal-Tits Magic Square.

\begin{tabular}{|l|l|l|l|}
\hline & & $C_{3,2}$ & $F_{4,4}$ \\
\hline & & $A_{5,2}$ & $E_{6,1}$ \\
\hline & $A_{5,3}$ & $D_{6,6}$ & $E_{7,7}$ \\
\hline & $E_{6,2}$ & $E_{7,1}$ & $E_{8,8}$ \\
\hline
\end{tabular}

\subsection{The general principle and some easy examples}

There are many possible definitions for what in general an equator geometry should be. We shall here give a practical definition that is easy to apply in different situations.

The starting point is the fact that in many Lie incidence geometries $\Delta$ the residue of a point is not only a quotient geometry, but it is also a subgeometry of $\Delta$, which can be seen as follows.

Proposition 4.1 Let $p$ be a point of a Lie incidence geometry $\Delta$ of rank at least 3 containing planes. Let $X$ be an object opposite $p$. Then on each line L through $p$ there is a unique point $p_{L}$ not opposite $X$. The set of such $p_{L}$, with lines inherited from the planes through $p$, is a Lie incidence geometry of the residue of $p$ in the building associated with $\Delta$.

For a simple Lie incidence geometry, there is only one case where it is of rank at least 3 and does not contain planes and this corresponds to the dual polar spaces. In this case, the residue can still be recovered as a subgeometry by considering the same point set as above, but by defining the line set as the set of so-called hyperbolic lines contained in it.

We generalise the above situation and define in general a trace geometry. 
Definition 4.2 Let $\Delta$ be a Lie incidence geometry related to a building $\mathfrak{B}$, say of rank $n$ and with type set $I$; more exactly let $\Delta$ be the $K$-Grassmannian of $\mathfrak{B}, K \subseteq I$. Let $F_{1}$ and $F_{2}$ be two opposite flags of $\mathfrak{B}$, say of common type $J$. Let $F$ be a flag of type, say, $J^{\prime}$ incident with $F_{1}$ and suppose that the projection of $F_{2}$ onto $F$ contains a point $p_{F}$. Then the set $T\left(F_{1}, F_{2} ; J^{\prime}\right)$ of points $p_{F}$, for $F$ ranging over the set of all flags of type $J^{\prime}$ incident with $F_{1}$ is called a $\left(J, J^{\prime}\right)$-trace in $\Delta$. Let $J^{*}$ be the union of $J$ with the type sets of the connected components of $I \backslash J$ disjoint from $J^{\prime}$. Then $T\left(F_{1}, F_{2} ; J^{\prime}\right)$ is obviously in natural bijective correspondence with the point set of the Lie incidence geometry $\Delta^{\prime}$ of the $J^{*}$-residue $\mathfrak{B}^{\prime}$ of $\mathfrak{B}$ corresponding to the $J^{\prime}$-Grassmannian. Endowing $T\left(F_{1}, F_{2} ; J^{\prime}\right)$ with the lines inherited from $\Delta^{\prime}$ turns $T\left(F_{1}, F_{2}, J^{\prime}\right)$ into a Lie incidence geometry, called the $\left(J, J^{\prime}\right)_{K}$-trace geometry. If we want a nontrivial set of lines, we must require $\left|J^{*}\right|<n-1$.

In many cases, the lines inherited from $\Delta^{\prime}$ are just the lines of $\Delta$ contained (as point sets) in $T\left(F_{1}, F_{2} ; J^{\prime}\right)$. We will see a prominent counter example below.

So, trace geometries in Lie incidence geometries are in fact either subgeometries, or point sets endowed with a set of abstract lines turning them into Lie incidence geometries. The most satisfying (and also most interesting) situation occurs when $F_{1}$ and $F_{2}$ play the same role, i.e., when $T\left(F_{1}, F_{2} ; J^{\prime}\right)=T\left(F_{2}, F_{1} ; J^{\prime}\right)$.

Definition 4.3 A trace geometry with point set $T\left(F_{1}, F_{2} ; J^{\prime}\right)$ is called an equator geometry if $T\left(F_{1}, F_{2} ; J^{\prime}\right)=T\left(F_{2}, F_{1} ; J^{\prime}\right)$. We talk about the $\left(J, J^{\prime}\right)_{K}$-equator geometry. If $J=K$, then we omit the subscript $K$ and write $\left(J, J^{\prime}\right)$-equator geometry.

In fact, one can be slightly more general and not require that $F_{1}$ and $F_{2}$ have the same type. But we do not insist on that since in all our examples they have the same type.

A convenient situation is where $F_{1}$ and $F_{2}$ are just points of $\Delta$, i.e., $J=K$. We now present some examples related to polar spaces. We start with the standard example.

Example 4.4 Let $\Delta$ be a polar space of rank $n$, hence a Lie incidence geometry of relative type $\mathrm{B}_{\mathrm{n}, 1}(k)$ or absolute type $\mathrm{D}_{\mathrm{n}, 1}(k)$. Let $J=\{1\}$ and $J^{\prime}=\{2\}$. Then $J^{*}=J$. Let $p_{1}$ and $p_{2}$ be two opposite points. Then $T\left(p_{1}, p_{2} ; J^{\prime}\right)$ is the set of points obtained by intersecting a line through $p_{1}$ with a line through $p_{2}$, hence $T\left(p_{1}, p_{2} ; J^{\prime}\right)=p_{1}^{\perp} \cap p_{2}^{\perp}=T\left(p_{2}, p_{1} ; J^{\prime}\right)$. The corresponding $(1,2)$-equator geometry is a polar space of rank $n-1$ and its lines are precisely the lines of $\Delta$ contained in $p_{1}^{\perp} \cap p_{2}^{\perp}$.

Example 4.5 Let $\Delta$ again be a polar space of rank $n$, over the type set $I$. Let $j \in I$ with $j \leq n-2$. Put $J=\{i\}$ and $J^{\prime}=\{i+1\}$. Then $J^{*}=\{1,2, \ldots, i\}$. Then the corresponding trace geometry is again an equator geometry coinciding with the subspace with point set $U_{1}^{\perp} \cap U_{2}^{\perp}$ for singular subspaces $U_{1}$ and $U_{2}$ of projective dimension $i-1$.

Example 4.6 Now let $\Delta$ be the Lie incidence geometry $\mathrm{B}_{\mathrm{n}, \mathrm{i}}(k), i \geq 1, n \geq 2 i$, or any Lie incidence geometry obtained from the $i$-Grassmannian of a polar space of rank $n$ at least $2 i$. Set $J=\{i\}$ and $J^{\prime}=\{2 i\}$. Then the $\left(J, J^{\prime}\right)$-trace geometry is an equator geometry and isomorphic to $\mathrm{B}_{\mathrm{n}-\mathrm{i}, \mathrm{i}}(k)$, or the Lie incidence geometry obtained from the $i$-Grassmannian of a polar space of rank $n-i$, respectively.

The case $i=2$ in the previous example is a long root geometry. More generally, we have the following proposition. 
Proposition 4.7 Let $\Delta$ be a long root geometry $\mathrm{X}_{\mathrm{n}, \mathrm{J}}(k)$, with $n \geq 4$. Let $\mathrm{Y}_{\mathrm{m}}$ be the connected diagram of longest length $m$ when removing the vertices of types $J$ from the Dynkin diagram $\mathrm{X}_{\mathrm{n}}$. Let $J^{\prime}$ be such that $\mathrm{Y}_{\mathrm{m}, J^{\prime}}(k)$ is a long root geometry. Then there is a $\left(J, J^{\prime}\right)$-equator geometry and it is isomorphic to $\mathrm{Y}_{\mathrm{m}, J^{\prime}}(k)$.

The proposition is easily proved noting that long root geometries are so-called hexagonal geometries; we do not go into detail here.

We now concentrate on some exceptional types. First we note that Proposition 4.7 gives us the following examples: The $(8,1)$-equator geometry of $\mathrm{E}_{8,8}(k)$ is $\mathrm{E}_{7,1}(k)$; The $(1,6)$-equator geometry of $\mathrm{E}_{7,1}(k)$ is $\mathrm{D}_{6,2}(k)$; The $(2,\{1,6\})$-equator geometry of $\mathrm{E}_{6,2}(k)$ is $A_{5,\{1,5\}}(k)$; The $(1,4)$-equator geometry of $\mathrm{F}_{4,1}(k)$ is $\mathrm{C}_{3,1}(k)$. Note that only in the latter case, the lines of the equator geometry are not induced by the lines of the larger Lie incidence geometry.

\subsection{Two examples related to $\mathrm{F}_{4}$}

Since the diagram of type $\mathrm{F}_{4}$ is symmetric, the Lie incidence geometry $\mathrm{F}_{4,4}(k)$ is also a hexagonal geometry, and so we also expect a $(4,1)$-equator geometry here. In fact, this equator geometry, which is isomorphic to $\mathrm{B}_{3,1}(k)$ (and note it is not a long root geometry) is extensively studied by De Schepper, Sastry \&Van Maldeghem [3]. We will use it in the next section for a combinatorial construction of $\mathrm{E}_{6,1}(k)$. We content ourselves here with mentioning that the lines of this $(4,1)$-equator geometry are hyperbolic lines in the symplecta, which are symplectic polar spaces of rank 3. Such hyperbolic lines are determined by any pair of its points, unlike the situation in the $(1,4)$-equator geometry.

A second example is the $(1,3)_{4}$-equator geometry in $\mathrm{F}_{4,4}(k)$. It is isomorphic to $\mathrm{C}_{3,2}(k)$.

Note that the same can be done with any metasymplectic space.

\subsection{Examples related to $E_{6}$}

First we consider the Lie incidence geometry $E_{6,1}(k)$. Let $J=\{2\}, J^{\prime}=\{5\}$. The corresponding $(2,5)_{1}$-equator geometry is $A_{5,2}(k)$. This is exactly the Lie incidence geometry appearing in the cell next to $\mathrm{E}_{6,1}(k)$. It is well-known that the latter can be viewed as a "projective plane" over the split octonions over $k$ and the former as a "projective plane" over de split quaternions over $k$. The embedding of $A_{5,2}(k)$ into $\mathrm{E}_{6,1}(k)$ as an equator geometry is the geometric evidence for the algebraic inclusion of the split quaternions in the split octonions.

We can go one step down and consider the Lie incidence geometry $A_{5,2}(k)$, set $J=$ $\{3\}$ and $J^{\prime}=\{1,4\}$. Then $J^{*}=J$ and the corresponding $(3,\{1,4\})_{2}$-equator geometry is $\mathrm{A}_{2,1}(k) \times \mathrm{A}_{2,1}(k)$, the Segre geometry $\mathscr{S}_{2,2}(k)$. This witnesses the inclusion of the ring $k \times k$ (with componentwise addition and multiplication) into the split quaternion algebra over $k$.

In the two cases considered in this subsection, we can characterize the equator geometries in a seemingly different way as follows (and the proofs are exercises in parapolar spaces).

Proposition 4.8 (i) The $(2,5)_{1}$-equator geometry of $\mathrm{E}_{6,1}(k)$ with respect to the opposite 5-spaces $W$ and $W^{\prime}$ of $\mathrm{E}_{6,1}(k)$ consists of the set of points of $\mathrm{E}_{6,1}(k)$ collinear with all points of a 3-space of $W$ and also with all points of a 3-space of $W^{\prime}$. 
(ii) The $(3,\{1,4\})_{2}$-equator geometry of $A_{5,2}(k)$ with respect to the opposite planes $U$ and $U^{\prime}$ of $\mathrm{E}_{5,2}(k)$ consists of the set of points of $\mathrm{A}_{5,2}(k)$ collinear with all points of a line of $U$ and also with all points of a line of $U^{\prime}$.

Next we fix $J=\{2\}$, and we list some examples $\mathrm{E}_{6, K}(k)$ for which there exists $J^{\prime}$ such that the $\left(J, J^{\prime}\right)_{K}$-equator geometry $\mathrm{A}_{5, J^{\prime \prime}}(k)$ exists.

\begin{tabular}{c|c|c}
$\mathrm{E}_{6, K}$ & $\left(J, J^{\prime}\right)_{K}$ & $\mathrm{~A}_{5, J^{\prime \prime}}$ \\
\hline \hline $\mathrm{E}_{6,1}$ & $(2,3)_{1}$ & $\mathrm{~A}_{5,2}$ \\
\hline $\mathrm{E}_{6,2}$ & $(2,\{1,6\})_{2}$ & $\mathrm{~A}_{5,\{1,5\}}$ \\
\hline $\mathrm{E}_{6,3}$ & $(2,1)_{3}$ & $\mathrm{~A}_{5,1}$ \\
& $(2,\{1,4\})_{3}$ & $\mathrm{~A}_{5,\{1,3\}}$ \\
\hline $\mathrm{E}_{6,4}$ & $(2,4)_{4}$ & $\mathrm{~A}_{5,3}$ \\
& $(2,\{1,5\})_{4}$ & $\mathrm{~A}_{5,\{1,4\}}$ \\
\hline $\mathrm{E}_{6,\{1,6\}}$ & $(2,\{3,5\})_{\{1,6\}}$ & $\mathrm{A}_{5,\{2,4\}}$
\end{tabular}

\subsection{Examples related to $E_{7}$}

There is no $J^{\prime} \subseteq\{1,2, \ldots, 7\}$ such that there is a $\left(7, J^{\prime}\right)$-equator geometry. However, in order to see $\mathrm{D}_{6,6}(k)$ as a subgeometry of $E_{7,7}(k)$, we set $J=\{1\}$ and $J^{\prime}=\{2\}$. Then the $(1,2)_{7}$-equator geometry exists and is actually isomorphic to $D_{6,6}(k)$. Again, this is geometric evidence for the algebraic inclusion of the split quaternions in the split octonions. To see the geometric evidence of the inclusion of the product of the field $k$ with itself (split quadratic extension) in the split quaternion algebra over $k$, we need to look at the $(5,3)_{6^{-}}$ equator geometry of $D_{6,6}(k)$, which is isomorphic to $A_{5,3}(k)$.

Finally, we see the inclusion of the Lie incidence and long root geometry $\mathrm{E}_{6,2}(k)$ in $\mathrm{E}_{7,1}(k)$ through the $(7,2)_{1}$-equator geometry of the latter.

\subsection{Examples related to $E_{8}$}

There are equator geometries in $\mathrm{E}_{8,8}(k)$ for $J=\{i\}$ any endpoint of the diagram, i.e., for all $i \in\{1,2,8\}$. We already mentioned the $(8,1)$-equator geometry, giving the geometric evidence $\mathrm{E}_{7,1}(k) \subseteq \mathrm{E}_{8,8}(k)$ of the fourth row of the Freudenthal-Tits Magic Square for the inclusion of the split quaternions in the split octonions over $k$. For $i=2$, we have the $(2,\{1,8\})_{8}$-equator geometry, isomorphic to $A_{7,\{1,7\}}(k)$. Finally, for $i=1$, we have the $(1,3)_{8}$-equator geometry giving rise to $D_{7,2}(k)$. Note that all these equator geometries are actually long root geometries.

\section{Projective Constructions}

In this section, we aim at constructing the $\mathrm{E}_{6,1}(k)$-variety in $\mathrm{PG}(26, k)$, for an arbitrary field $k$, in a purely geometric way.

To that aim, we first present an obviously but deliberately complicated construction of a Segre variety, proceed with a warming-up example and then explain the case of $\mathrm{E}_{6,1}(k)$. These three Lie incidence geometries are related to following three cells on the second row of the Freudenthal-Tits Magic Square. 


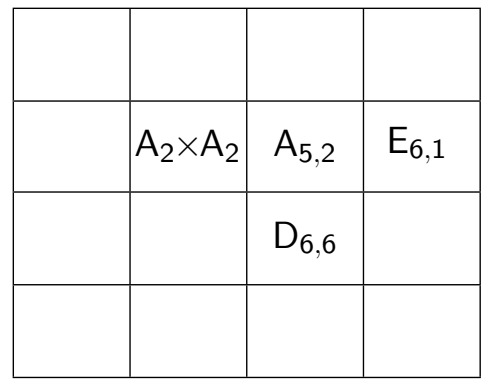

We also construct the half spin Lie incidence geometries in an inductive manner. This corresponds to the third cell in the third row above.

We begin with the latter.

\subsection{Hyperbolic polar spaces and half spin geometries}

In this section we will consider many isomorphisms between two structures; we will always assume that an isomorphism $\varphi$ acts on both structures and is involutive.

The following is a well known construction of the Lie incidence geometry $D_{2,2}(k)$, otherwise known under the name "ruled quadric in PG $(3, k)$ ".

Example 5.1 Consider two skew lines $L_{1}$ and $L_{2}$ in $\operatorname{PG}(3, k)$. Let $\varphi$ be a projectivity between them, i.e., $\varphi$ preserves the cross ratio of quadruples of points on $L_{i}, i=1,2$. Then the union of the lines $p p^{\varphi}$ with $p$ ranging over $L_{1}$ is a hyperbolic quadric.

This is a special case of the following straight forward construction.

Proposition 5.2 Let $U_{1}$ and $U_{2}$ be two disjoint subspaces of dimension $n-1$ in the projective space $\mathrm{PG}(2 n-1, k)$, and let $\varphi$ be a linear duality between $U_{1}$ and $U_{2}$, i.e., $\varphi$ maps points of $U_{i}$ onto $(n-2)$-spaces in $U_{i^{\prime}}$ such that collinear points are mapped onto concurrent subspaces in a bijective way preserving the cross ratio. Then the union of all lines $p_{1} p_{2}$, with $p_{1} \in U_{1}$ and $p_{2} \in p_{1}^{\varphi}$ is the point set of a hyperbolic quadric, i.e., the Lie incidence geometry $\mathrm{D}_{\mathrm{n}, 1}(k)$.

Proof Let $Q$ be any hyperbolic quadric in $\operatorname{PG}(2 n-1, k)$, and let $U_{1}$ and $U_{2}$ be two disjoint maximal singular subspaces, i.e., $U_{1}$ and $U_{2}$ are two disjoint $(n-1)$-space completely contained in $Q$. Let $p \in Q \backslash U_{2}$ be arbitrary and let $U \ni p$ be the unique maximal singular subspace of $Q$ intersecting $U_{2}$ in an $(n-2)$-space. Then, since the parity of the sum of the dimensions of the intersection of a maximal singular subpace with two given maximal singular subspaces is always constant, $U$ intersects $U_{1}$ in a point $p_{1}$. Hence $p$ is contained in the line $p_{1} p_{2}$, with $p_{2} \in p_{1}^{\perp} \cap U_{2}$. Now the mapping $\theta: U_{1} \rightarrow U_{2}^{*}: p_{1} \mapsto p_{1}^{\perp} \cap U_{2}$, with $U_{2}^{*}$ the dual space of $U_{2}$, is a linear duality. Indeed, we can take for $Q$ the quadric with equation $X_{-1} X_{1}+X_{-2} X_{2}+\cdots+X_{-n} X_{n}=0$, for $U_{1}$ the space with trivial positively indexed coordinates, and $U_{2}$ the space with trivial negatively indexed coordinates. Then $\theta$ maps the point $\left(x_{-1}, x_{-2}, \ldots, x_{-n}, 0,0, \ldots, 0\right)$ to the subspace with equations

$$
X_{-1}=X_{-2}=\cdots=X_{-n}=x_{-1} X_{1}+x_{-2} X_{2}+\cdots+x_{-n} X_{n}=0 .
$$

This defines $\varphi$ completely and uniquely and shows that it is a linear duality. 
Interestingly, also the half spin geometries can be constructed in a similar fashion, but this time starting with two half spin geometries of lower rank. Let us first demonstrate this in some low ranks.

The Lie incidence geometry $D_{3,3}(k)$ is just the projective space $\mathrm{PG}(3, k)$ viewed as point-line geometry. Application of the construction in Proposition 5.2 produces $D_{4,1}(k)$, which is isomorphic to the half spin geometry $\mathrm{D}_{4,4}(k)$ via triality. Now we consider the Lie incidence geometry $\mathrm{D}_{5,5}(k)$, corresponding to a hyperbolic quadric $Q$ in $\mathrm{PG}(9, k)$. Each point $p$ of $Q$ defines via its residue a unique subspace $Q_{p}$ of $\mathrm{D}_{5,5}(k)$ isomorphic to $\mathrm{D}_{4,1}(k)$. If $p$ and $q$ are non-collinear points, then there is no maximal singular subspace containing both, hence $Q_{p}$ and $Q_{q}$ are disjoint in this case. So let $p, q$ be non-collinear points of $Q$ and consider an arbitrary point of $D_{5,5}(k)$, i.e., a maximal singular subspace $U$ of $Q$ of certain prescribed type, say Type I and call the other type Type II. Assume that $p$ and $q$ do not belong to $U$. Then $W:=p^{\perp} \cap q^{\perp} \cap U$ is a singular subspace of dimension $n-3$ and hence defines a unique line $L$ of $D_{5,5}(k)$ containing $U,\langle p, W\rangle$ and $\langle q, W\rangle$. Hence every point of $\mathrm{D}_{5,5}(k)$ not contained in $q_{p} \cap Q_{q}$ lies on a unique line that intersects both $Q_{p}$ and $Q_{q}$ nontrivially. Hence, if we embed $Q_{p}$ and $Q_{q}$ in disjoint 7-dimensional subspaces of $\mathrm{PG}(15, k)$, then $\mathrm{D}_{5,5}(k)$ is contained in $\mathrm{PG}(15, k)$. Now suppose $U$ belongs to $Q_{p}$. Then all points of $Q_{q}$ that are incident with $\left\langle q, q^{\perp} \cap U\right\rangle$ (the latter viewed as a-singular-subspace of $Q_{q}$ ) are collinear with $U$ in $\mathrm{D}_{5,5}(k)$. Hence $\mathrm{D}_{5,5}(k)$ is the union of all lines $p^{*} q^{*}$, where $p \in Q_{p}$ and $q^{*} \in Q_{q}$ such that $q^{*} \in\left(p^{*}\right)^{\varphi}$ for some linear duality $\varphi$ between $Q_{p}$ and $Q_{q}$.

A similar argument proves the following construction result. First note that, by taking the residue of an element of type $n-2$ in a building of type $\mathrm{D}_{n}$, we see that the structure of the set of maximal subspaces of $\mathrm{D}_{n, n}(k), n \geq 4$ (maximal subspaces have dimension $n-1-$ those corresponding to the elements of type $n-1$ of the corresponding building - and 3 - corresponding to the elements of type $n-3$ of the corresponding building), containing a fixed line of $\mathrm{D}_{n, n}(k)$ is a Segre variety $\mathscr{S}_{n-3,1}(k)$. Hence it makes sense to talk about the cross ratio of a quadruple of disjoint maximal subspaces of maximal dimension.

Proposition 5.3 Let $Q_{1}$ and $Q_{2}$ be two half spin geometries isomorphic to $\mathrm{D}_{\mathrm{n}, \mathrm{n}}(k), n \geq 4$, embedded in disjoint subspaces of dimension $2^{n-1}-1$ of $\mathrm{PG}\left(2^{n}-1, k\right)$. Let $\varphi$ be any linear duality between $Q_{1}$ and $Q_{2}$ (i.e., $\varphi$ maps (collinear) points to maximal singular subspace intersecting in a line and preserves the cross ratio). Then the union of all lines $p_{1} p_{2}$, with $p_{1} \in Q_{1}$ and $p_{2} \in p^{\varphi}$ is an embedding of the Lie incidence geometry $D_{\mathrm{n}+1, \mathrm{n}+1}(k)$.

Using an obvious induction argument, this construction easily implies that we are dealing here with the universal embedding.

\subsection{Two constructions of the Segre variety $\mathscr{S}_{2,2}(k)$}

Let $\pi_{1}, \pi_{2}, \pi_{3}, \pi_{4}$ be four planes in $\mathrm{PG}(8, k)$ such that no three of them are contained in a hyperplane. Then every point $x$ of $\pi_{1}$ is contained in a unique plane $\pi_{x}$ of PG $(8, k)$ intersecting all of $\pi_{2}, \pi_{3}, \pi_{4}$ in points. The union of all such planes (for $x$ ranging over $\pi_{1}$ ) is the Segre variety $\mathscr{S}_{2,2}(k)$.

For the second construction we remind the reader of the following property of $\mathscr{S}_{2,2}(k)$, proved by Schillewaert \& Van Maldeghem in [5] .

Proposition 5.4 (i) Every pair of points of $\mathscr{S}_{2,2}(k)$ not contained in a common plane of $\mathscr{S}_{2,2}(k)$ is contained in a unique subgeometry isomorphic to $\mathscr{S}_{1,1}(k)$. 
(ii) Every pair subgeometries isomorphic to $\mathscr{S}_{1,1}(k)$ of $\mathscr{S}_{2,2}(k)$ intersect in either a point or a line.

Let $p$ be a point of PG $(8, k)$, and let $\pi_{1}, \pi_{2}$ be two planes of $\mathrm{PG}(8, k)$ intersecting in $\{p\}$. Let $Q$ be some hyperbolic quadric in a 3-space disjoint from $\left\langle\pi_{1}, \pi_{2}\right\rangle$. If the configuration $\pi_{1} \cup \pi_{2} \cup Q$ is to be part of a Segre variety $\mathscr{S}_{2,2}(k)$, then each line of $Q$ is contained in a plane of $\mathscr{S}_{2,2}(k)$ that intersects $\pi_{1} \cup \pi_{2}$ in a unique point. Moreover, by the existence of sub-Segre varieties isomorphic to $\mathscr{S}_{1,1}(k)$ (which are just hyperbolic quadrics in 3-spaces), we see that these points constitute two lines, one line $L_{1}$ in $\pi_{1}$ and a line $L_{2}$ in $\pi_{2}$. Let $\mathscr{R}_{1}$ and $\mathscr{R}_{2}$ be the two reguli of $Q$. Each of these has the natural structure of a projective line over $k$. Then indices can be chosen such that $\mathscr{S}_{2,2}(k)$ defines projectivities $\theta_{i}: \mathscr{R}_{i} \rightarrow L_{i}$ with the property that the planes spanned by $R \in \mathscr{R}_{i}$ and $R^{\theta_{i}}$ belong to $\mathscr{S}_{2,2}(k), i=1,2$.

Adding these planes to our data $\pi_{1}, \pi_{2}, Q$ does not yet determine $\mathscr{S}_{2,2}(k)$ uniquely. Note, however, that all these data are independent and can be chosen freely.

Now, by Proposition 5.4 we know that every point of $\mathscr{S}_{2,2}(k)$ outside $\pi_{1} \cup \pi_{2}$ is contained in a unique hyperbolic quadric $Q_{q}$ containing $p$ and intersecting $Q$ in a unique point $q$. Let $M_{i}, i=1,2$ be defined as $q \in M_{i} \in \mathscr{R}_{i}$. Set $x_{i}=M_{i}^{\theta_{i}}, i=1,2$. Then the lines $\left\langle p x_{1}\right\rangle$, $\left\langle p, x_{2}\right\rangle,\left\langle q x_{1}\right\rangle$ and $\left\langle q, x_{2}\right\rangle$ are four lines of $Q_{q}$. We claim that fixing the quadric $Q_{q}$ for one point $q$ determines $\mathscr{S}_{2,2}(k)$ uniquely.

Indeed, fix $q \in Q$. Then, following Example 5.1, and with the notation of the previous paragraph, $Q_{q}$ is determined by a projectivity $\varphi:\left\langle p, x_{1}\right\rangle \rightarrow\left\langle q, x_{2}\right\rangle$. Now let $q^{\prime}$ be any other point of $Q$. By connectivity of $Q$, we may assume that $\left\langle q, q^{\prime}\right\rangle$ is a generator of $Q$, and without loss we can assume it belongs to $\mathscr{R}_{1}$. Let $M_{2}^{\prime} \in \mathscr{R}_{2}$ be such that $q^{\prime} \in M_{2}^{\prime}$ and set $x_{2}^{\prime}=M_{2}^{\prime \theta_{2}}$. Then the planes $\left\langle q, q^{\prime}, x_{1}\right\rangle$ and $\pi_{2}$ belong to $\mathscr{S}_{2,2}(k)$ and the three lines $\left\langle p, x_{1}\right\rangle$, $\left\langle q, x_{2}\right\rangle$ and $\left\langle q^{\prime}, x_{2}^{\prime}\right\rangle$ also belong to $\mathscr{S}_{2,2}(k)$ and each meet each of these planes. It follows that, for an arbitrary point $x \in\left\langle p, x_{1}\right\rangle$, the plane $\pi_{x}$ of $\mathscr{S}_{2,2}(k)$ containing the line $\left\langle x, x^{\varphi}\right\rangle$ also intersects $\left\langle q^{\prime}, x_{2}^{\prime}\right\rangle$. It follows that $\pi_{x}$ contains the line $K$ distinct from $\left\langle q, x_{2}\right\rangle$ belonging to the quadric of $\mathscr{S}_{2,2}(k)$ determined by $L_{2}$ and $M_{1}$ and $\theta_{2}$. In this quadric, $K$ is determined by a projectivity $\phi:\left\langle q, x_{2}\right\rangle \rightarrow\left\langle q^{\prime}, x_{2}^{\prime}\right\rangle$. We conclude that $Q_{x}$ is determined by the projectivity $\varphi^{\prime}:\left\langle p, x_{1}\right\rangle \rightarrow\left\langle q^{\prime}, x_{2}^{\prime}\right\rangle: x \mapsto x^{\varphi \phi}$.

Hence $\mathscr{S}_{2,2}(k)$ is completely determined by $\pi_{1}, \pi_{2}, L_{1}, L_{2}, Q, \theta_{1}, \theta_{2}$ and $\varphi$.

\subsection{A line Grassmannian}

We now construct $A_{5,2}(k)$ in a similar fashion. This is a warming up for the next subsection where we will construct $\mathrm{E}_{6,1}(k)$.

We again start with a point $p$ in $\mathrm{PG}(14, k)$. Instead of the lines $L_{1}, L_{2}$, we now take the local structure of $A_{5,2}(k)$, which is a Segre variety $\mathscr{S}_{1,3}(k)$. So we consider a 7-space $U_{1}$ in $\mathrm{PG}(14, k)$ not containing $p$ and three mutually disjoint 3 -spaces in $U_{1}$. Let $\mathfrak{S}$ be the Segre variety consisting of all lines intersecting all of these three 3 -spaces in points. We call these lines the line-generators of $\mathfrak{S}$. The family $\mathscr{F}$ of all line-generators naturally has the structure of a projective 3-space (by intersection with an arbitrary 3-space intersecting all line-generators), which we denote by $\Pi$. Let $\mathfrak{C}$ be the cone with vertex $p$ and base $\mathfrak{S}$. Let $U_{2}$ be a 5-space disjoint from $\left\langle p, U_{1}\right\rangle$ and consider a hyperbolic quadric (a Klein quadric) $Q$ in $U_{2}$. Let $\mathscr{R}_{i}, i=1,2$, be the two systems of generators of $Q$.

Since $Q$ is Lie incidence geometry $\mathrm{D}_{3,1}(k)=\mathrm{A}_{3,2}(k)$, the family $\mathscr{R}_{1}$ is the point set of a 3 -space, and hence there is a natural isomorphism $\theta: \mathscr{F} \rightarrow \mathscr{R}_{1}$. We can choose $\theta$ linear, 
i.e., such that it preserves the cross ratio. For each line $L \in \mathscr{F}$, we add the 4 -space $\left\langle L, L^{\theta}\right\rangle$ to our data.

As in the previous subsection, it now remains to add, for each point $q \in Q$, a unique Klein quadric $\mathscr{K}_{q}$ containing $p$ and $q$. Fix $q \in Q$. The inverse image of $q$ under $\theta$ is a line of $\Pi$, hence a hyperbolic quadric $\mathscr{H}$ in $\mathfrak{S}$. Fix two arbitrary points $a, b$ of $\mathscr{H}$, not on a common generator. Let $L$ and $M$ be disjoint generators of $\mathscr{H}$ containing $a$ and $b$, respectively. Then the planes $\alpha:=\langle p, L\rangle$ and $\beta:=\langle q, M\rangle$ are opposite planes in $\mathscr{K}_{q}$ and so, in view of Proposition 5.2, $\mathscr{K}_{q}$ is completely determined by a linear duality $\delta$ between $\alpha$ and $\beta$. For each point $x$ on $L$, there is a unique point $y \in M$ such that $\langle x, y\rangle$ is a generator of $\mathscr{H}$. Hence $x^{\delta}=\langle q, y\rangle$. Moreover, $p^{\delta}=M$. Hence it suffices to know the image of one more point in $\alpha \backslash(L \cup\{p\})$. We claim that this is enough to complete the construction of $\mathrm{A}_{5,2}(k)$. Indeed, it suffices to prove that any point $q^{\prime} \in Q$ can be included in a Segre geometry isomorphic to $\mathscr{S}_{2,2}(k)$ containing $p$ and $q$, because then we can apply the construction in Subsection 5.2.

Clearly there exist opposite points $x_{1}$ and $x_{2}$ in $Q$ collinear to both $q$ and $q^{\prime}$. Let $L_{i}$ be a line of $\mathfrak{S}$ all of whose points are collinear to $x_{i}, i=1,2$, with $L_{1}$ and $L_{2}$ contained in distinct 3 -spaces of $\mathfrak{S}$. Since $q$ and $q^{\prime}$ are collinear to $x_{1}$ and $x_{2}$, both $q$ and $q^{\prime}$ are collinear with unique points of both $L_{1}$ and $L_{2}$. It follows that $q$ and $q^{\prime}$ are collinear with all points of unique lines in the planes $\left\langle x_{1}, L_{1}\right\rangle$ and $\left\langle x_{2}, L_{2}\right\rangle$. Since also $p$ is collinear with the points of unique lines in these planes (namely, $L_{1}$ and $L_{2}$ ), we deduce from Proposition 4.8(ii) that $p, q, q^{\prime}$ are contained in a Segre geometry isomorphic to $\mathscr{T}_{2,2}(k)$.

\subsection{The Cartan variety}

A construction of $\mathrm{E}_{6,1}(k)$ similarly to the previous subsection exists by considering a cone with vertex some point $p$ and base a half spin geometry $\mathfrak{D}$ isomorphic to $D_{5,5}(k)$ as constructed in Proposition 5.3 (this cone spans a 16-dimensional subspace $U_{1}$ of $\mathrm{PG}(26, k)$ ) and a hyperbolic quadric $Q$ in a complementary 9-space $U_{2}$. We can identify each point of $\mathfrak{D}$ with a certain 4 -space of $Q$, and we can do so in a linear way, i.e., respecting the cross ratio inherited from $U_{1}$ and $U_{2}$. If we identify a point $x$ with the 4-space $W$, then we add the 5-space $\langle x, W\rangle$ to our data. Then a point $q$ of $Q$ is collinear to the points of a hyperbolic quadric $Q_{q}$ on $\mathfrak{D}$ isomorphic to $\mathrm{D}_{4,1}(k)$. So we have two cones $\mathfrak{C}_{p}$ and $\mathfrak{C}_{q}$ with vertex $p$ and $q$, respectively, and with base $Q_{q}$. Again, $p, q$ and $Q_{q}$ define a unique hyperbolic quadric isomorphic to $\mathrm{D}_{5,1}(k)$ if we know for one point $a \in \mathfrak{C}_{p} \backslash\left(Q_{q} \cup\{p\}\right)$ a collinear point $b \in \mathfrak{C}_{q} \backslash\left(Q_{q} \cup\{q\}\right)$. Fixing such a collinear pair determines all others, just as in the previous subsection (now using Proposition 4.8(i) to construct an equator geometry isomorphic to $\left.A_{5,2}(k)\right)$.

\section{Combinatorial Constructions}

In this section, we construct bigger Lie incidence geometries out of smaller ones. Our main goal is here to report on the construction of $\mathrm{E}_{6,1}(k)$ out of $\mathrm{F}_{4,4}(k)$. In view of the Freudenthal-Tits Magic Square, there is an analogue for $A_{5,2}(k)$ out of $C_{3,2}(k)$. We will also explain this baby example. This means that we cover the following cells of the square. 


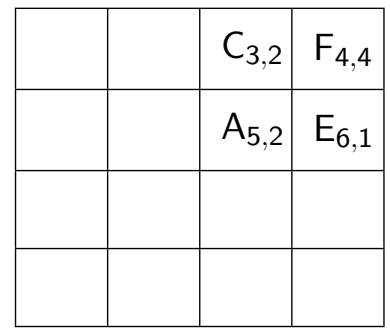

We also briefly mention similar phenomena for other Lie incidence geometries. We begin with the latter.

\subsection{Obvious examples}

Example 6.1 Let $C_{n, 1}$ be the natural Lie incidence geometry related to a symplectic polar space, $n \geq 2$. For two non-collinear points $p, q$ we define the set $L_{p, q}=\left(p^{\perp} \cap q^{\perp}\right)^{\perp}$. Obviously $p, q \in L_{p, q}$, and it turns out that $L_{p, q}=L_{p^{\prime}, q^{\prime}}$, for all $p^{\prime}, q^{\prime} \in L_{p . q}$. If we add all sets $L_{p, q}$, for $p, q$ ranging through the set of points, with $p$ not collinear to $q$, to the geometry $\mathrm{C}_{\mathrm{n}, 1}$ as additional lines, then we obtain $A_{2 n-1,1}(k)$, the ordinary $(2 n-1)$-dimensional projective space over $k$.

Example 6.2 Similarly as the previous example, we can construct the half-spin geometry $\mathrm{D}_{\mathrm{n}+1, \mathrm{n}+1}(k)$ from the dual polar space $\mathrm{B}_{\mathrm{n}, \mathrm{n}}(k), n \geq 2$ (for $n=2$, this is also the case $n=2$ of Example 6.1).

Example 6.3 Let $\mathrm{G}_{2,1}(k)$ be the Lie incidence geometry also known under the name of split Cayley hexagon. Let $p$ and $q$ be two opposite points and let $L_{p, q}$ be the set of points collinear with $p$ and not opposite $q$. Then, adding these sets, for all choices of $p$ and $q$, to the set of lines of $\mathrm{G}_{2,1}(k)$ produces $\mathrm{B}_{3,1}(k)$.

These examples all have the property that the point sets of the two geometries are the same, and only new lines need to be defined. In the next two examples we also have to extend the point set.

\subsection{The line Grassmannian $A_{5,2}(k)$ again}

Let $\Delta$ be the Lie incidence geometry $\mathrm{C}_{3,2}(k)$. Then $\Delta$ is a hexagonal parapolar space with symplecta isomorphic to $C_{2,1}(k)$, the symplectic quadrangle over $k$. Define the set $\mathscr{H}$ as the set of hyperbolic lines of $\Delta$, where a hyperbolic line is a set $L_{p, q}=\left(p^{\perp} \cap q^{\perp}\right)^{\perp}$ in some symplecton $\Sigma$ (see Example 6.1), for some non-collinear points $p, q$ in $\Sigma$. If we add $\mathscr{H}$ to the line set of $\Delta$, we obtain a geometry $\Delta^{\prime}$ of diameter 2 . The convex closure in $\Delta^{\prime}$ of two lines that are opposite in $\Delta$ is a geometry isomorphic to $\mathrm{B}_{2,1}(k)=\mathrm{C}_{2,2}(k)$ and is called a pseudo symplecton. Let $\mathfrak{G}$ be such a geometry, and let $x$ be an arbitrary point in $\mathfrak{G}$. Select an arbitrary point $y$ of $\mathfrak{G}$ not $\Delta^{\prime}$-collinear with $x$. Let $L$ be any line of $\Delta$ through $y$. Since $x$ and $y$ are opposite in $\Delta$, there is a unique point $z$ on $L$ not opposite $x$ and there is a unique point $u \Delta$-collinear with both $x$ and $z$. The set of pseudo symplecta defined by $x$ and some point on $L \backslash\{z\}$ is called a pencil of pseudo symplecta. One can show that it only depends on $u$ and one member of it (hence independent of $L$ ). We call $u$ the (unique) centre of the pencil. 
We now define new points as the pseudo symplecta, and new lines are the pencils of pseudo symplecta together with their centre.

The geometry of new and old points, and of old lines, hyperbolic lines and new lines is isomorphic to the Lie incidence geometry $A_{5,2}(k)$.

\subsection{Metasymplectic spaces and $\mathrm{E}_{6,1}(k)$}

Now we construct $\mathrm{E}_{6,1}(k)$ out of the (split) metasymplectic space $\mathrm{F}_{4,4}(k)$. This construction prominently uses the $(4,1)$-equator geometries of $\mathrm{F}_{4,4}(k)$.

So we start with $\mathrm{F}_{4,4}(k)$. Our goal is to add two new kinds of lines and one new kind of points, just as in the previous subsection. One new kind of lines is given by the family of hyperbolic lines in the subgeometries isomorphic to $\mathrm{C}_{3,1}(k)$ (residues of elements of type 4). The new points are the extended equator geometries. We now explain what this is.

Consider two opposite points $p, q$ and let $\mathfrak{E}_{p, q}$ be the corresponding $(4,1)$-equator geometry. We take the union $\widetilde{\mathfrak{E}}$ of all equator geometries $\mathfrak{E}_{x, y}$, for $\{x, y\}$ ranging over the pairs of opposite points in $\mathfrak{E}_{p, q}$. Endowed with all hyperbolic lines in it, $\widetilde{\mathfrak{E}}$ is a Lie incidence geometry isomorphic $\mathrm{B}_{4,1}(k)$, called an extended equator geometry. The family of all extended equator geometries is the set of new points.

The last type of lines is not so difficult to define. Consider an extended equator geometry $\widetilde{\mathfrak{E}}$ and a maximal singular subspace $U$ in there. Recall that $U$ is a set of points of $\mathrm{F}_{4,4}(k)$ such that the hyperbolic lines contained in it render it the point-line geometry $\mathrm{A}_{4,1}(k)$ of a projective space of dimension 4 over the field $k$. One shows that there is a unique point $x$ collinear in $\mathrm{F}_{4,4}(k)$ with all points of $U$. Then the set of all extended equator geometries containing $U$, together with the point $x$, is a generic new line.

One now shows that the geometry of old and new points, and of old, hyperbolic and new lines is isomorphic to $\mathrm{E}_{6,1}(k)$, see De Schepper, Sastry \& Van Maldeghem [3].

\section{Coxeter Complexes and associated Graphs}

Let $(W, S)$ be a spherical Coxeter system and $\Delta(W, S)=(X, \Omega)$ the corresponding Coxeter complex. We define $\Gamma(W, S)$ as the graph with set of vertices $X$ and two vertices are adjacent if they form a respective chamber with the same panel. It follows that $\Gamma(W, S)$ has as many connected components as its rank is. Each node of the corresponding Coxeter diagram defines a connected component by considering the vertices of $\Delta(W, S)$ of that particular type. If the node is numbered $i$, then we denote the corresponding connected graph by $\Gamma_{i}(W, S)$. If $(W, S)$ corresponds to the spherical diagram $\mathrm{X}_{n}$ of rank $n$, then $\Gamma_{i}(W, S)$ is precisely the thin Lie incidence geometry $\mathrm{X}_{n, i}(1)$, if we identify each edge with a (thin) line. We shall also view $\mathrm{X}_{n, i}(1)$ as a graph.

In many cases the automorphism group of $\Gamma_{i}(W, S)$ is precisely $W$. Prominent counter examples are $\Gamma_{i}(W, S)$ for $(W, S)$ of type $\mathrm{D}_{n}$ and $1 \leq i \leq n-2$, since in these cases, the automorphism group clearly contains the Coxeter group of type $\mathrm{B}_{n}$. But this is only a special case of the general phenomenon that, if the node labelled $i$ is stable under a graph automorphism group of the Coxeter diagram, then the automorphism group of $\Gamma_{i}(W, S)$ also contains that graph automorphism group.

The graphs $\Gamma_{i}(W, S)$ are also standard apartments of the buildings of type $(W, S)$. In this respect a good description of the apartment helps one understand the geometry of the 
building. For instance, all possible mutual positions of certain flags can be deduced from those in an apartment.

As an example, the graphs $\mathrm{A}_{n, i}(1)$ are precisely the Johnson graphs $J(n+1, i)$ : the vertices are the $i$-subsets of an $(n+1)$-set, two such $i$-sets being adjacent if they intersect in an $(i-1)$-set.

We now concentrate on some constructions of apartments for the exceptional types $E_{6}$, $E_{7}, E_{8}$ and $F_{4}$, occasionally giving rise to similar constructions of apartments of certain classical types. The nicest constructions are those of $X_{n, \ell}(1)$, where $X \in\{E, F\}$ and $\ell$ is an end node of the diagram. Hence we will restrict to these cases. At the end, we will have covered the following cells of the Magic Square:

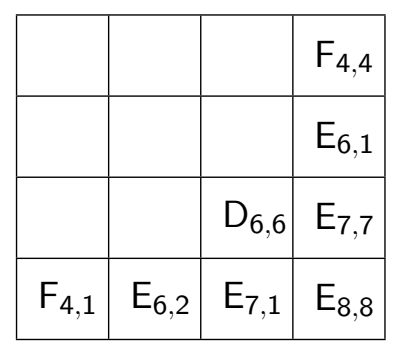

\subsection{Apartments of type $E_{6}$}

It is well known that the Coxeter group of type $E_{6}$ is isomorphic to the orthogonal group $\mathrm{O}_{6}^{-}(2)$, which in turn is isomorphic to the unitary group $\mathrm{U}_{5}(2)$. These groups are automorphism groups of the generalized quadrangle $Q(2,4)$. It is not a coincidence that $Q(2,4)$ and $\mathrm{E}_{6,1}(1)$ both have 27 points. In fact, the complement of the collinearity graph of $Q(2,4)$ is exactly the thin Lie incidence geometry $\mathrm{E}_{6,1}(1)$ considered as a graph. Example 2.9 implies the following construction of $\mathrm{E}_{6,1}(1)$.

Example 7.1 Let $N=\{1,2,3,4,5,6\}$ and $N^{\prime}=\left\{1^{\prime}, 2^{\prime}, 3^{\prime}, 4^{\prime}, 5^{\prime}, 6^{\prime}\right\}$. Set $V=N \cup N^{\prime} \cup\left(\begin{array}{c}N \\ 2\end{array}\right)$. Let adjacency be given by $a \sim b, a^{\prime} \sim b^{\prime}, a \sim a^{\prime}$ and $a \sim\{b, c\} \sim a^{\prime}$ and $\{a, b\} \sim\{a, c\}$, for all distinct $a, b, c \in N$.

The previous example can be written down in a more systematic and general way as follows. First note that the graphs induced on $N$ and $N^{\prime}$ are complete graphs, more exactly they are isomorphic to $A_{5,1}(1)$. The graph induced on $\left(\begin{array}{l}N \\ 2\end{array}\right)$ is isomorphic to $A_{5,2}(1)$. The labelling using $1,2, \ldots$ and $1^{\prime}, 2^{\prime}, \ldots$ boils down to the choice of an isomorphism between the various underlying $\mathrm{A}_{5}(1)$. To determine adjacency between vertices of $N$ and $N^{\prime}$ we use the chosen isomorphism and connect corresponding vertices. We say that adjacency is induced by natural isomorphism (with symbol $\cong$ ). A vertex $a$ of $N$ is adjacent to a vertex $\{b, c\}$ of $\left(\begin{array}{l}N \\ 2\end{array}\right)$ if $\{b, c\}$, as a line of $A_{5}(1)$ is incident to the hyperplane of $A_{5}(1)$ obtained from $a$ by applying the natural duality $x \leftrightarrow N \backslash\{x\}$. We say that adjacency is induced by natural duality (with symbol $\asymp$ ).

We picture this construction as follows. 


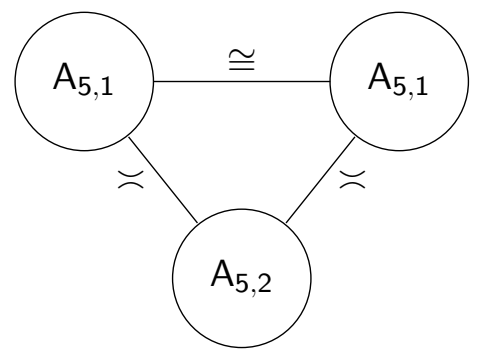

This way of writing the construction is of course not unique. One can try to use as many isomorphisms as possible, e.g., as follows.

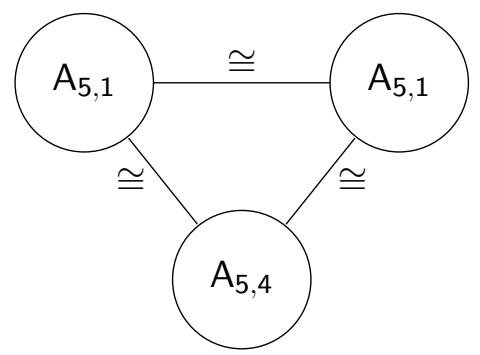

So this diagram means that we have three disjoint graphs, namely two copies of $A_{5,1}(1)$, and a copy of $A_{5,4}(1)$. We choose a standard thin building of type $A_{5}$, which we fix by defining the set of elements of type 1 as $\{a, b, c, d, e, f\}$. Then the vertices of each copy of $\mathrm{A}_{5,1}(1)$ are precisely $a, b, c, d, e, f$, and the vertices of the graph $\mathrm{A}_{5,4}(1)$ are all 4-subsets of $\{a, b, c, d, e, f\}$. Adjacency between an element $x \in\{a, b, c, d, e, f\}$ of a copy of $\mathrm{A}_{5,1}(1)$ and an element $X \subseteq\{a, b, c, d, e, f\},|X|=4$, of $A_{5,4}(1)$ is given by $x \in X$; adjacency between an element $x \in\{a, b, c, d, e, f\}$ of one copy of $A_{5,1}(1)$ and an element $y \in\{a, b, c, d, e, f\}$ of another copy of $A_{5,1}(1)$ is given by $x=y$.

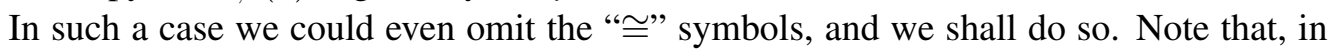
this thin geometry, it is easy to see that $A_{5,1}(1)$ is the $(2,1)_{1}$-trace geometry of $A_{5,2}(1)$ and $A_{5,2}(1)$ is the $(2,5)_{1}$-equator geometry of $E_{6,1}(1)$.

It is a common feature of the constructions of Coxeter complexes in this section that the different parts are trace and equator geometries. But there are also exceptions, at least if we do not consider a generalisation of the notion of trace geometry with respect to opposite flags of different type. An example is the following.

Example 7.2 Let $D_{5,1}(1)$ be the thin polar space of rank 5, i.e., the complete graph on the vertex set $\left\{1,2,3,4,5,1^{\prime}, 2^{\prime}, 3^{\prime}, 4^{\prime}, 5^{\prime}\right\}$ minus the matching $\left\{\left(1,1^{\prime}\right),\left(2,2^{\prime}\right),\left(3,3^{\prime}\right),\left(4,4^{\prime}\right),\left(5,5^{\prime}\right)\right\}$. Let $D_{5,5}(1)$ be the thin half spin geometry with vertex set the 5-cliques of $D_{5,1}(1)$ which intersect $\{1,2,3,4,5\}$ in an odd number of vertices, and adjacency intersecting in three vertices. Let $\Gamma$ be the graph with set of vertices a symbol $\infty$, the vertices of $D_{5,1}(1)$ and the vertices of $D_{5,5}(1)$, and define adjacency as follows. The vertex $\infty$ is adjacent to every vertex of $D_{5,5}(1)$; the adjacency inside the sets of vertices of $D_{5,1}(1)$ and $D_{5,5}(1)$ is the natural one; a vertex $x$ of $\mathrm{D}_{5,1}(1)$ is adjacent to a vertex $\{a, b, c, d, e\}$ of $\mathrm{D}_{5,5}(1)$ if $x \in\{a, b, c, d, e\}$. Then $\Gamma$ is isomorphic to $\mathrm{E}_{6,1}(1)$.

Again, we can consider the adjacency between $D_{5,1}(1)$ and $D_{5,5}(1)$ as incidence given by the natural isomorphism of the labelling of the points of $D_{5,1}(1)$. We picture this construction as follows. 


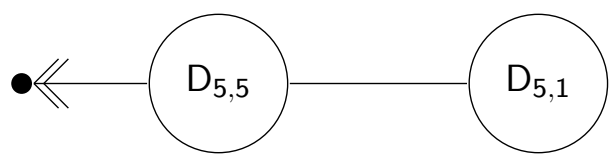

The construction in Subsecion 5.4 of $\mathrm{E}_{6,1}(k)$ is a "thickening" of the above construction of $E_{6,1}(1)$. Note also that the construction of $D_{5,1}(1)$ as a complete graph minus a matching is equivalent to the construction pictured as follows.

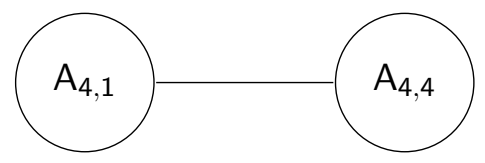

which is the thin version of the case $n=5$ of the construction given by Proposition 5.2. Generalisation to $\mathrm{D}_{n, 1}(1)$ is obvious. Likewise, the thin version of Proposition 5.3 is pictured as follows.

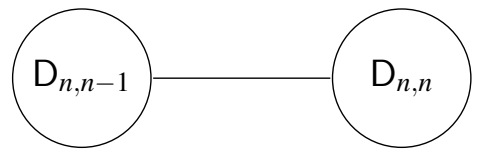

which provides a construction of $\mathrm{D}_{n+1, n+1}(1)$.

Now we turn to $\mathrm{E}_{6,2}(1)$. This is a thin long root geometry, and all thin long root geometries behave similarly. Hence the same pattern as we will see now will repeat itself for $E_{7,1}(1), E_{8,8}(1)$ and $F_{4,1}(1)$. Long root geometries have equator geometries, and this will show in the construction of the corresponding apartments.

The first construction is performed using trace and equator geometries with respect to two opposite vertices.

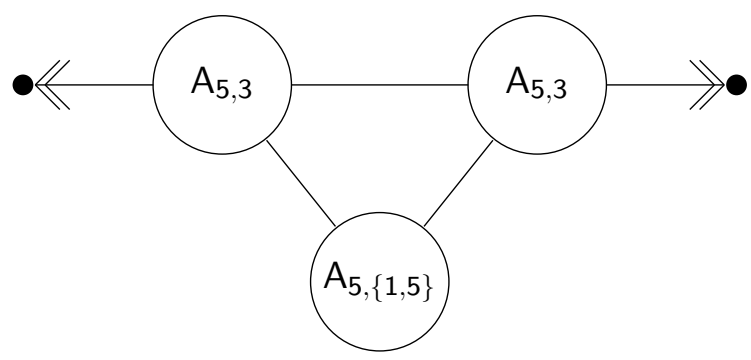

The next construction is performed using trace and equator geometries with respect to an opposite pair of half spin geometries of rank 4, i.e., opposite elements of type 1 and 6.

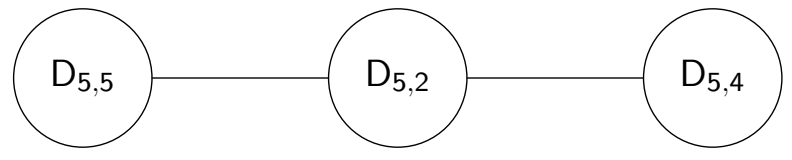

\subsection{Apartments of type $E_{7}$}

The thin Lie incidence geometry $E_{7,7}(1)$ is, still viewed as a graph, isomorphic to the Gosset graph. There are several constructions of this graph, but we will only mention those that fit in our approach. The first one is with respect to two opposite vertices. Then there are two trace geometries none of which is an equator geometry. 


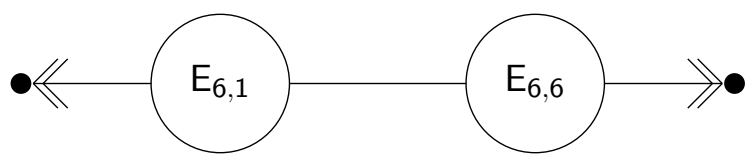

A similar construction exists for the thin Lie incidence geometry $D_{6,6}(1)$ (which is not coincidentally next to $E_{7,7}(1)$ in the Magic Square). It goes as follows.

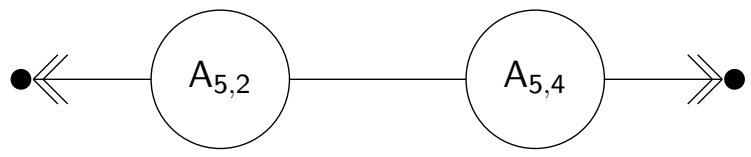

The second construction of $E_{7,7}(1)$ is with respect to two opposite elements of type 2, hence two subgeometries isomorphic to $A_{6,1}(1)$. This yields the following diagram.

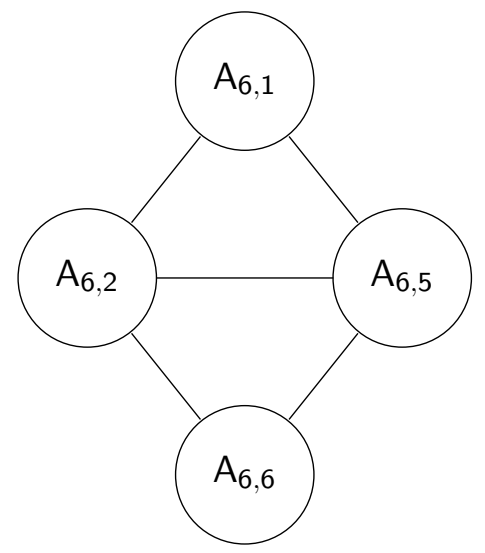

The next construction displays the $(1,2)_{7}$-equator geometry. It is based on two opposite subgeometries isomorphic to $D_{6,1}(1)$.

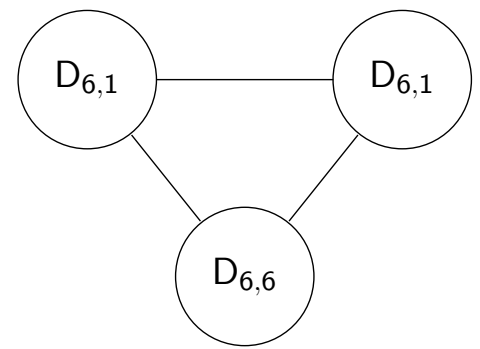

Now we consider $E_{7,1}(1)$, which is a long root geometry. The canonical construction is with respect to two opposite vertices, and we can read off the residue (as a trace geometry) and the equator geometry.

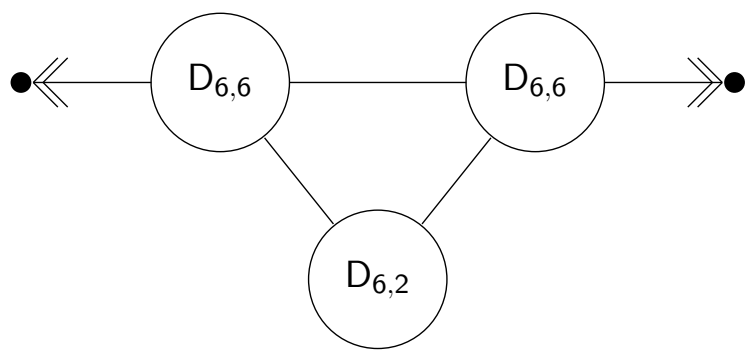


The second construction is with respect to two subgeometries isomorphic to $E_{6,1}(1)$. It reveals the $(7,2)_{1}$-equator geometry.

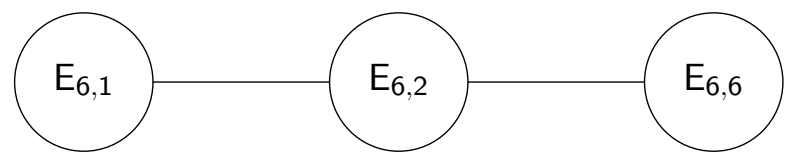

There is a striking similarity with the construction of $E_{6,2}(1)$ using $D_{5,5}(1)$ and $D_{5,2}(1)$, see above. We will encounter another case of the same shape below when discussing apartments of type $F_{4}$. Yet another example is given by a construction of $D_{6,6}(1)$, using two opposite thin projective 5-spaces, and it can be pictured as follows.

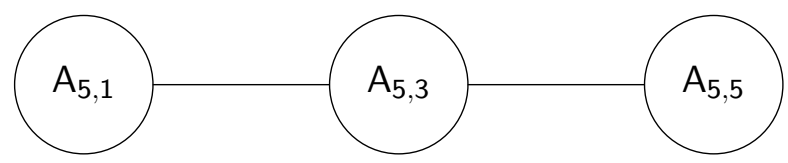

This construction can be used to derive the following, and third, construction of the thin long root geometry $\mathrm{E}_{7,1}(1)$.

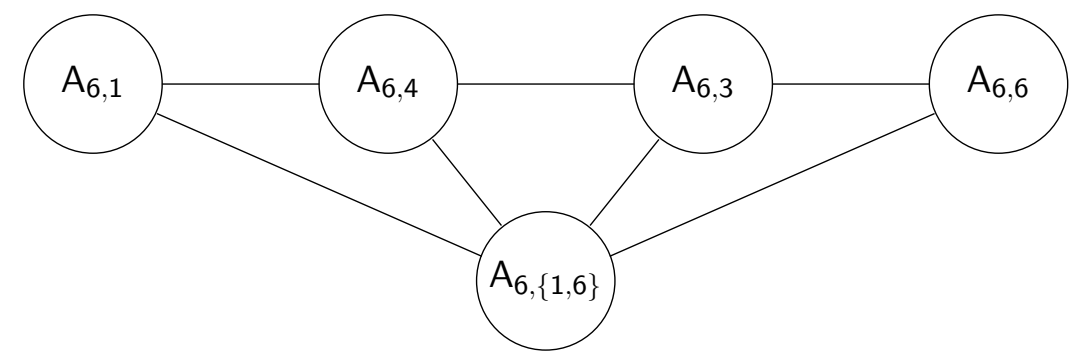

Note that this suggests that $A_{6,\{1,6\}}(k)$ be the $(2,\{1,7\})_{7}$-equator geometry of $E_{7}(k)$, for any field $k$.

Finally we would also like to mention a construction of $E_{7,2}$, which has 576 vertices and is therefore unpopular. We present the simplest one, using two opposite elements of type 7, i.e., two subgeometries isomorphic to a thin long root geometry of type $\mathrm{E}_{6}$.

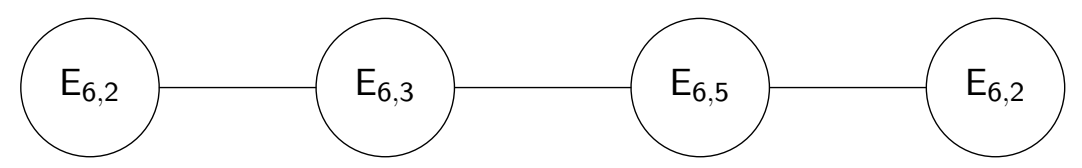

\subsection{Apartments of type $E_{8}$}

Here we mainly concentrate on $\mathrm{E}_{8,8}(1)$. We provide three constructions, one for each end node of the diagram.

We begin with respect to two opposite vertices (elements of type 8). Then we get the usual long root picture. 


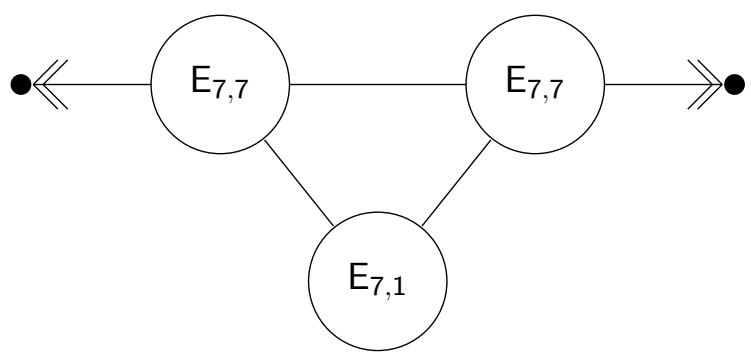

The second construction is with respect to two opposite elements of type 1, i.e., two opposite thin subgeometries isomorphic to $\mathrm{D}_{7,1}(1)$. It is similar to the third construction of $\mathrm{E}_{7,1}(1)$ and looks as follows.

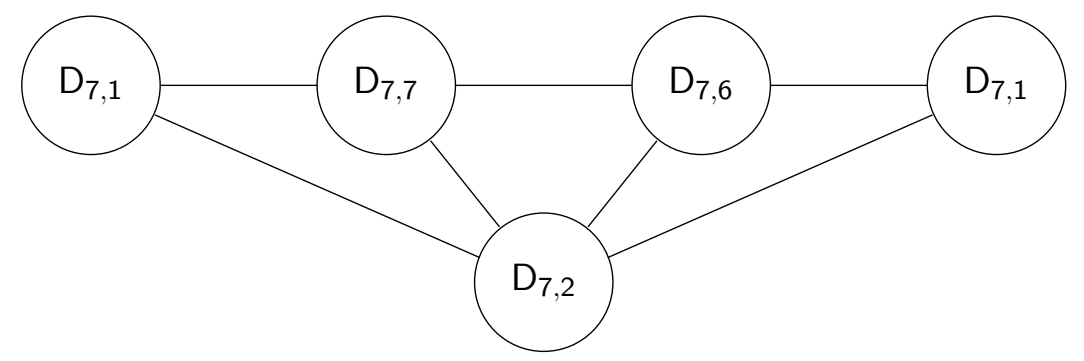

Finally, we construct $E_{8,8}(1)$ with respect to two opposite elements of type 2 . This gives the following remarkable construction, where all of $A_{7, i}(1)$ are used, $1 \leq i \leq 7$, except that $A_{7,4}(1)$ is replaced with the thin long root geometry $A_{7,\{1,7\}}(1)$.

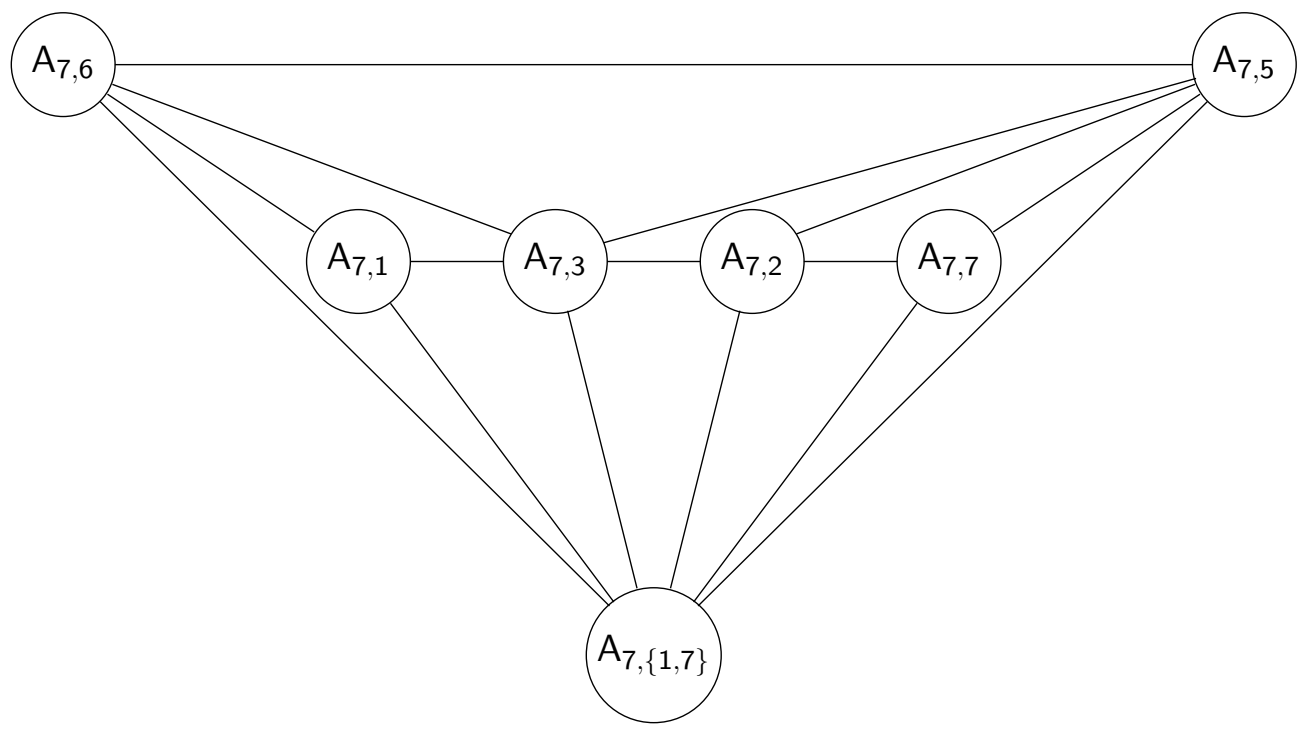

Note that this diagram is not entirely symmetric. There is indeed an isomorphism between $A_{7,3}(1)$ and $A_{7,5}(1)$, but no edges between $A_{7,2}(1)$ and $A_{7,6}(1)$ (unrelated detail: the latter keeps the graph in the picture above planar as otherwise it would contain a complete subgraph with the five vertices $A_{7,2}(1), A_{7,3}(1), A_{7,5}(1), A_{7,6}(1)$ and $A_{7,\{1,7\}}(1)$ ).

We cannot resist to also give a less familiar example, namely, a construction of $E_{8,1}(1)$. The Lie incidence geometry $\mathrm{E}_{8,1}(k)$, for a field $k$, turns up in several characterisation theorems of parapolar spaces, so the construction below gives some information about the structure of that geometry (see also Remark 7.4). 
We construct $E_{8,1}(1)$ with respect to two opposite elements of type 8, i.e., two opposite subgeometries isomorphic to $E_{7,1}(1)$. It has 2160 vertices and degree 64 .

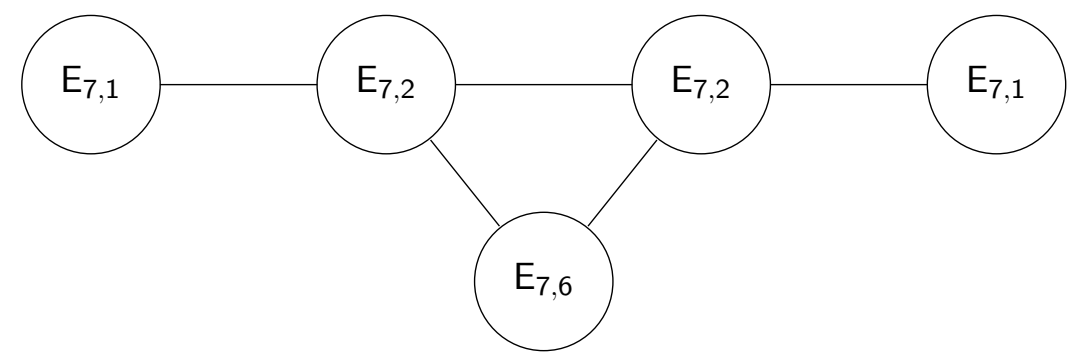

This suggests that $\mathrm{E}_{7,6}(k)$ is a $(1,6)_{8}$-equator geometry of $\mathrm{E}_{8}(k)$.

\subsection{Apartments of type $\mathrm{F}_{4}$}

This is the first Coxeter diagram with a double bond that we encounter. The two end nodes of the $\mathrm{F}_{4}$-diagram play the same role, except that the arrow on the double bond takes away the symmetric. The graphs $F_{4,1}(1)$ and $F_{4,4}(1)$ are nevertheless isomorphic. However, it is still more convenient to consider $F_{4,1}(1)$ because it corresponds to long root geometries.

There is a second special feature when dealing with Dynkin diagrams with double bonds. It concerns the long root geometries of buildings of type $\mathrm{B}_{n}, n \geq 2$, and $\mathrm{C}_{n}, n \geq 3$. The buildings of these types correspond to polar spaces. In general, one thinks of a polar space as a Lie incidence geometry of type $\mathrm{B}_{n, 1}$, and then the corresponding long root geometry has type $\mathrm{B}_{n, 2}$ and is a non-strong parapolar space of diameter 3 . However, strictly speaking, long root geometries are only well defined for split buildings, and when the polar space is a symplectic one-hence belonging to the diagram $\mathrm{C}_{n}$ - the long root geometry is the polar space itself. Now, all other long root geometries have diameter 3, except this one. We will correct this and view the long root geometry of a symplectic polar space as a geometry without lines, where points have distance 2 and 3 (points collinear in the polar space have distance 2, the others distance 3). Also, the lines of the polar space will be conceived as the symplecta of the long root geometry. In this setting, the thin long root geometry $C_{3,1}(1)$ is a edgeless graph with six vertices endowed with an opposition relation which is a matching.

This opposition relation allows to well define isomorphisms between such structures, and also incidence preserving maps to other Lie incidence geometries of the same main type. For example, if we label the vertices of the graph $\left\{1,2,3,1^{\prime}, 2^{\prime}, 3^{\prime}\right\}$ and declare $a$ opposite $a^{\prime}$, for all $a \in\{1,2,3\}$, then the point set of $\mathrm{C}_{3,3}(1)$ is, with self-explaining notation, equal to $\left\{123,1^{\prime} 23,12^{\prime} 3,123^{\prime}, 12^{\prime} 3^{\prime}, 1^{\prime} 23^{\prime}, 1^{\prime} 2^{\prime} 3,1^{\prime} 2^{\prime} 3^{\prime}\right\}$. The natural isomorphism then connects 1 with $123,123^{\prime}, 12^{\prime} 3$ and $12^{\prime} 3^{\prime}$, and it connects 123 with 1,2 and 3 . It is in this way that the below pictures must be read.

With these conventions, we have the following construction of $F_{4,1}(1)$. It is performed with respect to two opposite vertices, as a long root geometry. 


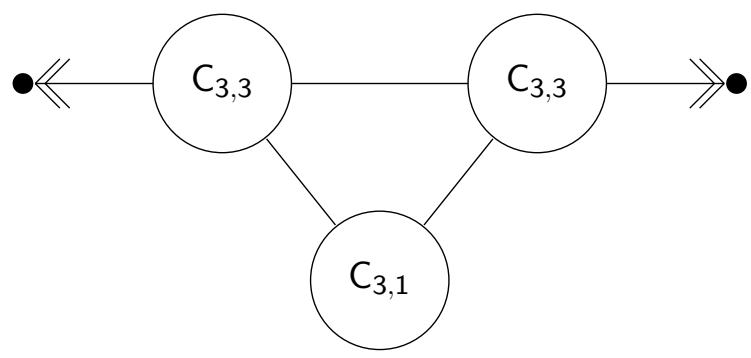

With respect to two opposite symplecta (elements of type 4), we have the following construction.

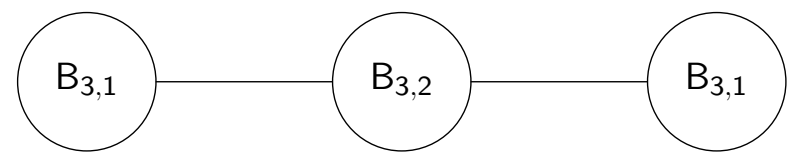

Here, $B_{3,2}(1)$ is the ordinary thin long root geometry of type $B_{3}$.

Remark The diagrams that we have drawn in this section act as a kind of floor plan for the corresponding Lie incidence geometry (to stay in the terminology of real estate). The different components are exactly the types of the trace geometries one can find in these Lie incidence geometries. Such a trace geometry is an equator geometry if and only if it lies symmetrically in the diagram. In theory, there is a floor plan for each Lie incidence geometry and each choice of types of opposite elements. The most interesting floor maps are those for which these opposite elements correspond to irreducible subbuildings (which is automatic when the types correspond to end nodes of the Coxeter diagram).

\section{Conclusion and open problems}

In this paper, we have presented a lot of combinatorial and geometric constructions of spherical buildings, focusing on the Lie incidence geometries related to the Freudenthal-Tits Magic Square. However, some cells of that square always remained empty. The full Square looks as follows.

\begin{tabular}{|l|l|l|l|}
\hline$A_{1,1}$ & $A_{2,\{1,2\}}$ & $C_{3,2}$ & $F_{4,4}$ \\
\hline$A_{2,1}$ & $A_{2} \times A_{2}$ & $A_{5,2}$ & $E_{6,1}$ \\
\hline$C_{3,3}$ & $A_{5,3}$ & $D_{6,6}$ & $E_{7,7}$ \\
\hline$F_{4,1}$ & $E_{6,2}$ & $E_{7,1}$ & $E_{8,8}$ \\
\hline
\end{tabular}

The cells that we left empty throughout the whole article contain $A_{1,1}, A_{2,\{1,2\}}, A_{2,1}$ and $C_{3,3}$. The first one is a rather trivial geometry consisting of points on a single line. Geometrically, it is convenient to think of it as a conic. The geometries of type $A_{2,\{1,2\}}$ are the flag complexes of a projective plane, i.e., non-thick generalized hexagons with thick lines. The representation they take in the Magic Square is related to triality of the hyperquadric of type 
$D_{4}$, although these only cover the case of characteristic 3 . These and the others can also be obtained by intersecting a Segre variety $\mathscr{S}_{2,2}(k)$ with an appropriate hyperplane. The geometries of type $A_{2,1}$ are ordinary projective planes, and the corresponding representation in the Magic Square are the ordinary Veronesean varieties, which are intersections of Segre varieties with appropriate subspaces. The same thing holds for the Lie incidence geometries $\mathrm{C}_{3,3}(k)$, which are symplectic dual polar spaces contained in $\mathrm{A}_{5,3}(k)$.

We now mention the most important open problems related to the constructions here presented.

1. Find an explicit set of quadrics in 77-, 132- and 247-dimensional projective space over the field $k$ whose intersection is the Lie-incidence (long root) geometry $\mathrm{E}_{6,2}(k)$, $\mathrm{E}_{7,1}(k)$ and $\mathrm{E}_{8,8}$, respectively.

2. Study and classify the inclusions of Lie incidence geometries.

3. Find projective constructions for the geometries in the third row of the FreudenthalTits Magic square.

Concerning the second one, it is tempting to conjecture that, perhaps under mild conditions, inclusions will always arise from trace (in particular, equator) geometries, if not entirely contained in a singular (projective) subspace.

\section{References}

[1] N. Bourbaki, Groupes et Algèbres de Lie, Chapters 4,5 and 6, Actu. Sci. Ind., 1337 Hermann, Paris (1968).

[2] A. M. Cohen, Point-line geometries related to buildings, Handbook of Incidence Geometry, Buildings and Foundations, (ed. F. Buekenhout), Chapter 9, North-Holland, Amsterdam (1995). pp. 647-737.

[3] A. De Schepper, N.S.N. Sastry \& H. Van Maldeghem, Split buildings of type $F_{4}$ in buldings of type $\mathrm{E}_{6}$, Abh. Math. Sem. Univ. Hamburg 88 (2018), 97-160.

[4] J. W. P. Hirschfeld \& J. A.Thas, General Galois Geometries, Oxford Mathematical Monographs, Oxford Science Publications, The Clarendon Press, Oxford University Press, New York (1991).

[5] J. Schillewaert \& H. Van Maldeghem, Projective planes over quadratic 2-dimensional algebras, Adv. Math. 262 (2014), 784-822.

[6] J. Tits, Sur certaines classes d'espaces homogènes de groupes de Lie,, Acad. Roy. Belg. Cl. Sci. Mém. Collect. $8^{\circ}$ (2), 29 (1955).

[7] J. Tits, Buildings of Spherical Type and Finite BN-Pairs, Springer Lecture Notes Series, 386 Springer-Verlag, Berlin-Heidelberg-New York (1974). 